\title{
Delta-like 4 is required for pulmonary vascular arborization and alveolarization in the developing lung
}

\author{
Sheng Xia, ${ }^{1}$ Heather L. Menden, ${ }^{1}$ Nick Townley, ${ }^{1}$ Sherry M. Mabry, ${ }^{1}$ Jeffrey Johnston, ${ }^{2}$ \\ Michael F. Nyp, ${ }^{1}$ Daniel P. Heruth, ${ }^{1}$ Thomas Korfhagen, ${ }^{3}$ and Venkatesh Sampath ${ }^{1}$ \\ 'Division of Neonatology, Department of Pediatrics, Children's Mercy Hospital, Kansas City, Missouri, USA. ${ }^{2}$ Genomic \\ Medicine Center, Children's Mercy Hospital, Kansas City, Missouri, USA. ${ }^{3}$ Division of Neonatology, Department of \\ Pediatrics, Cincinnati Children's Hospital, Cincinnati, Ohio, USA.
}

\begin{abstract}
The molecular mechanisms by which endothelial cells (ECs) regulate pulmonary vascularization and contribute to alveolar epithelial cell development during lung morphogenesis remain unknown. We tested the hypothesis that delta-like 4 (DLL4), an EC Notch ligand, is critical for alveolarization by combining lung mapping and functional studies in human tissue and DLL4haploinsufficient mice (DI/4+/lacz). DLL4 expressed in a PECAM-restricted manner in capillaries, arteries, and the alveolar septum from the canalicular to alveolar stage in mice and humans. DII4 haploinsufficiency resulted in exuberant, nondirectional vascular patterning at E17.5 and P6, followed by smaller capillaries and fewer intermediate blood vessels at P14. Vascular defects coincided with polarization of lung EC expression toward JAC1-NICD-HES1 signature and decreased tip cell-like (Car4) markers. DII4+/lacz mice had impaired terminal bronchiole development at the canalicular stage and impaired alveolarization upon lung maturity. We discovered that alveolar type I cell (Aqp5) markers progressively decreased in $\mathrm{DII}^{+/ / a c z}$ mice after birth. Moreover, in human lung EC, DLL4 deficiency programmed a hypersprouting angiogenic phenotype cell autonomously. In conclusion, DLL4 is expressed from the canalicular to alveolar stage in mice and humans, and DII4 haploinsufficiency programs dysmorphic microvascularization, impairing alveolarization. Our study reveals an obligate role for DLL4-regulated angiogenesis in distal lung morphogenesis.
\end{abstract}

Authorship note: SX and HLM contributed equally to this work.

Conflict of interest: The authors have declared that no conflict of interest exists.

Copyright: (c) 2021, Xia et al. This is an open access article published under the terms of the Creative Commons Attribution 4.0 International License.

Submitted: October 9, 2019

Accepted: February 25, 2021

Published: April 8, 2021

Reference information: JCI Insight 2021:6(7):e134170.

https://doi.org/10.1172/jci.

insight.134170.

\section{Introduction}

Mammalian lung development progresses sequentially through the embryonic, pseudoglandular, canalicular, saccular, and alveolar phases (1). In preterm infants, exposure to noxious stimuli, such as infection, mechanical ventilation, and hyperoxia, disrupts lung development, resulting in bronchopulmonary dysplasia (BPD) $(2,3)$. Vascular maldevelopment is characterized by defects in pulmonary vascular growth and dysmorphic arborization, both of which are hallmarks of $\operatorname{BPD}(4,5)$. The molecular mechanisms regulating distal lung vascularization, a requisite for alveolarization, are not fully understood, limiting our understanding of defective vascularization in BPD. Vasculogenesis and angiogenesis are distinct mechanisms that govern vascular network development. Based on vascular casts and electron microscopy of mouse lungs demonstrating initial discontinuity, followed by fusion of proximal and distal lung vasculature, deMello et al. suggested that vasculogenesis is important for peripheral pulmonary blood vessel development $(6,7)$ Parera et al. mapped the endothelial cell-specific Tie2 promoter during lung development and suggested that angiogenesis is required for distal lung vascular arborization (8). However, the functional contribution of angiogenesis or vasculogenesis to lung vascular development and alveolarization $(1,9)$ has not been investigated. Moreover, the key endothelial cell (EC) molecular players that regulate angiogenesis and pulmonary vascular arborization required for normal alveolar development remain unknown.

During sprouting angiogenesis, expression of delta-like 4 (DLL4) in EC, induced by a VEGF gradient specifies a tip cell EC phenotype. Tip cells specify a stalk cell EC phenotype in adjoining EC, through transactivation of NOTCH-dependent signaling that inhibits DLL4 expression (10). Both homozygous and heterozygous deficiency of Dll4 result in embryonic lethality between E9.5 and E11.5 in C57BL/6 mice with the absence of large vitelline vessels in yolk sac and reduced dorsal 
aorta $(11,12)$. During retinal vascular development, EC-specific Dll4 deficiency leads to upregulation of tip cell-enriched genes, followed by more tip cells and vessel branches (13), whereas more DLL4 expression represses sprouting angiogenesis, EC proliferation, and migration (14). Besides angiogenesis, D114/Notch signaling maintains arterial identity of blood vessels, with Dll4 deficiency reducing arterial vascular lumen diameter in embryos $(11,15)$. Although these studies evince a critical role for DLL4 in embryonal and retinal vascular development, the contribution of DLL4 to postnatal organ development, specifically lung vascularization and alveolarization, remains unknown.

Although the contribution of VEGF to lung development is established, the EC-specific molecular mechanisms that regulate distal lung vascularization and alveolarization have yet to be characterized (1618). Based on our initial studies showing DLL4 expression in the mouse and human lung we hypothesized that DLL4 is required for pulmonary microvascular growth and arborization during distal lung morphogenesis, and Dll4 deficiency will impair alveolarization. In this study, we used Dll4 ${ }^{+/ l a c z}$ mice (CD1 strain) lacking 1 copy of Dll4 to investigate the contribution of angiogenesis to lung development, and determine whether impaired DLL4-dependent angiogenesis disrupts alveolarization. Our data suggest that Dll4 haploinsufficiency programs deviant angiogenesis and altered alveolar type I cell development, resulting in dysmorphic vascularization and disrupted alveolarization.

\section{Results}

DLL4 is expressed during mouse and human lung ontogeny. To determine whether DLL4 is important for lung vascularization we mapped DLL4 in canalicular (C57BL/6 mouse $[\mathrm{M}]-\mathrm{E} 17.5$, human $[\mathrm{H}]-22$ weeks gestational age [GA]), saccular (M: P2; H: 27-week GA), and alveolar (M: P7, P14, and P21; H: 36- and 39-week GA) stages in mouse (C57BL/6) and human autopsy samples. In the human and mouse lungs, DLL4 (red) was expressed in a PECAM-restricted manner (green) from the canalicular phase (Figure 1, A and B). DLL4 expression was scant in the canalicular phase, increased in the saccular phase (P2), and was widely distributed in the early, middle, and late alveolar phase (Figure 1A). Similar patterns of DLL4 expression were found in cadaveric human lung sections obtained from the late canalicular (22 week), saccular (27 week), and early alveolar (36 and 39 week) stages (Figure 1B). To confirm DLL4 antibody staining, we performed X-gal staining on frozen lung sections of Dll4 ${ }^{+/ l a c z}$ CD1 pups, where 1 Dll4 allele is replaced by the lac $Z$ reporter (11). LacZ expression was sparse in the canalicular stage, but showed abundant expression during postnatal lung development (Figure 2A), consistent with our mapping studies in C57BL/6 mice and human autopsy specimens. Costaining X-gal (blue) with EPHB4 (brown, Figure 2B) and PECAM (brown, Figure 2C), respectively, showed that Dll4 is expressed in an EC-restricted manner in branching capillaries and arteries, but not in veins (Figure 2, B and C).

We next quantified temporal changes in Dll4 RNA in mouse lungs at E17.5, P4, P14, and P28 to determine whether postnatal increase in pulmonary microvascular arborization coincides with changes in Dll4 expression. Dll4 mRNA expression increased exponentially from canalicular stage to saccular phase and remained elevated through the alveolarization stage (Figure $2 \mathrm{D}$ ), with $50 \%$ reduction in $\mathrm{Dll4}^{+/ l a c Z}$ mice (Figure 2D). During angiogenesis, EC tip cells, enriched in DLL4, lead sprouting angiogenesis, and stalk cells follow the tip cell. DLL4 expression in the leading EC was observed with X-gal/PECAM IHC staining in $D_{l l 4^{+/ l a c Z}}$ mouse lungs (Figure 2C), and with DLL4/PECAM immunofluorescent (IF) staining in human and C57BL/ 6 mouse lung samples (see below). Our data from human samples and from 2 mouse strains (CD1, C57BL/6) demonstrate that Dll4 is expressed during lung ontogeny from the canalicular phase, supporting its role in pulmonary vascular development.

Dll4 predominantly expresses in lung EC. To confirm that Dll4 expression is predominantly restricted to EC in the lung, we applied single-cell RNA-Seq (scRNA-Seq) in P14 C57BL/6 mouse lungs $(n=3)$. We identified 4 major groups of cells in 5677 cells pooled from 3 mice using established marker genes (19): epithelial cells (Epcam and Cdh1), ECs (Pecam and Cdh5), stromal cells (Colla1 and colla2), and immune cells (Ptprc), which included DCs, macrophages, B cells, T cells, basophils, and neutrophils (Figure 3A). We next generated Dll4 expression plots, which showed that Dll4 is mainly expressed in EC (Figure 3A). To verify scRNA-Seq results, we stained PECAM (EC marker), PDGFRA (myofibroblast marker), AQP5 (AT1 marker), and SPC (AT2 marker) with X-gal or anti- $\beta$-gal costaining separately. X-gal and PDGFRA IHC staining indicated that PDGFRA ${ }^{+}$fibroblasts did not express Dll4 (Figure 3B). Additionally, $\beta$-gal and AQP5/SPC IF staining showed that Dll4 did not colocalize with AT1 and AT2 cells (Figure 3C). However, DLL4/PECAM IF staining (Figure 1, A and B) and X-gal/PECAM IHC staining (Figure 2C) revealed 
A

PECAM

DLL4
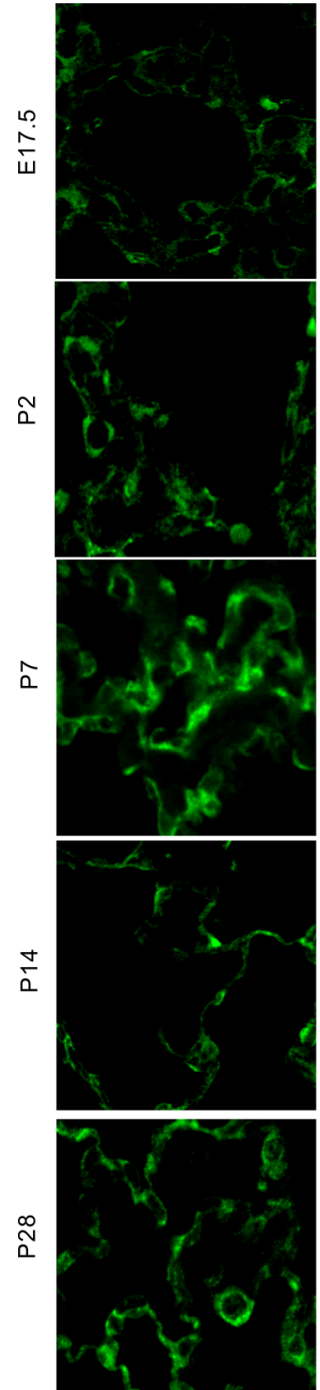
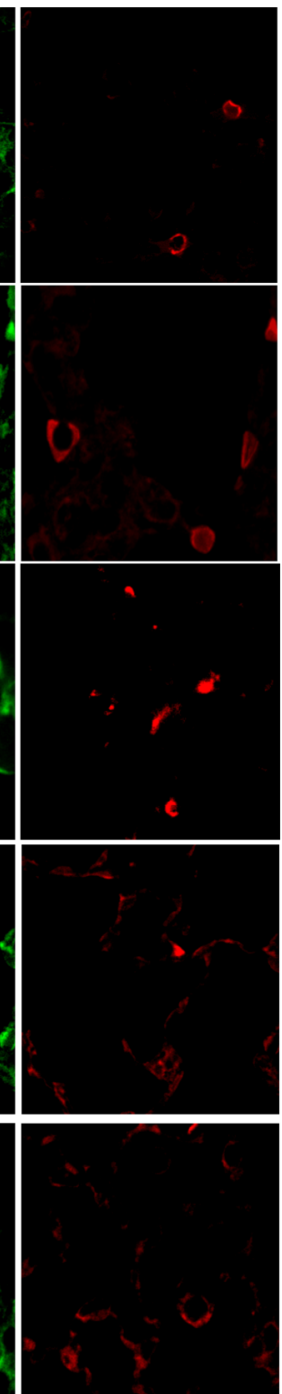

Merge
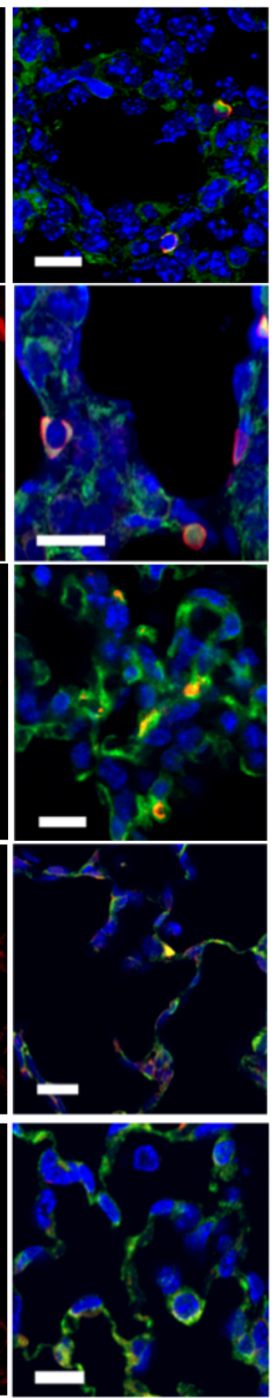

B

PECAM
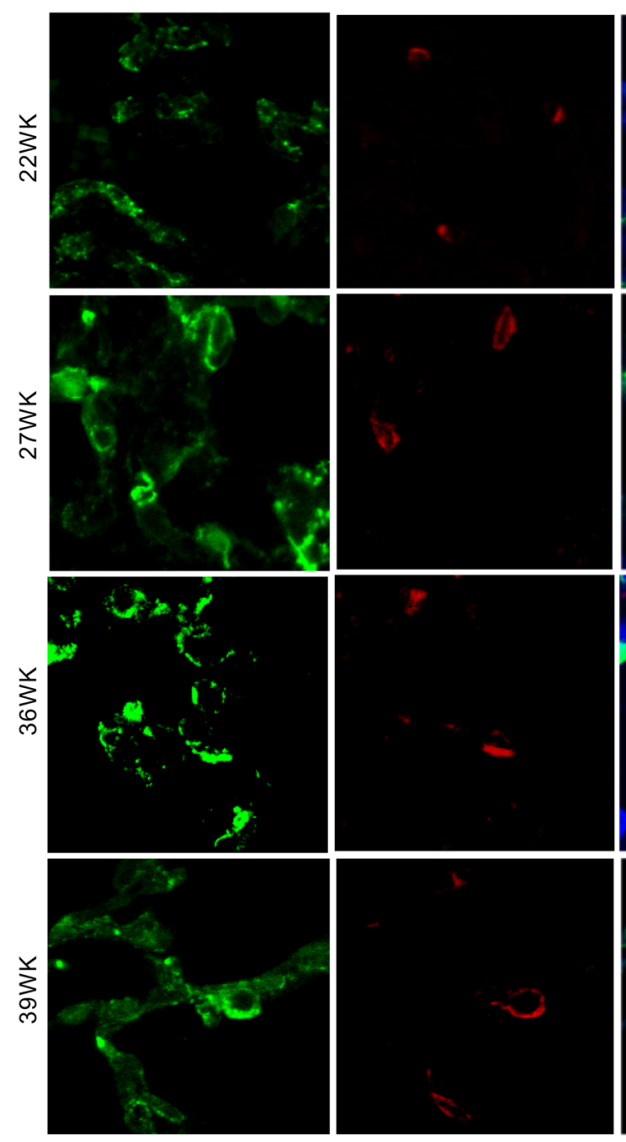

Merge
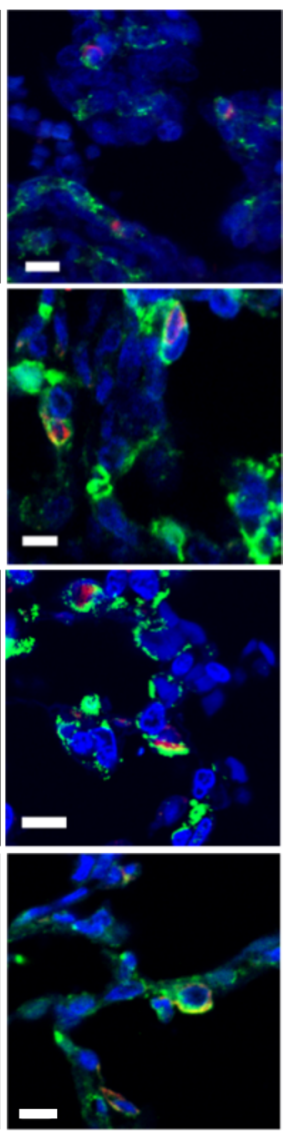

Figure 1. DLL4 is expressed from the canalicular through alveolar stage of mouse and human lung development. (A) DLL4 (red), PECAM (green), and nucleus (DAPI, blue) staining on mouse lung samples at E17.5, P2, P7, P14, and P28. E17.5 represents canalicular stage; P2 represents saccular stage; and P7, P14, and P28 represent early, middle, and completed alveolar stage. (B) DLL4 (red) and PECAM (green) staining on human lung slides at 22, 27, 36, and 39 gestational age weeks (22, 27, and 36/39 week represent human canalicular, saccular, and early alveolar stages). Scale bars: $10 \mu \mathrm{m}$. $n=4$ per group.

that Dll4 expressed in EC. Our scRNA-Seq and immunostaining data demonstrate that Dll4 is prominently expressed in pulmonary EC.

Altered expression of EC genes that specify EC fate in Dll4 ${ }^{+/ l a c Z}$ mice. We compared gene expression that specifies EC fate during sprouting angiogenesis between $D l l 4^{+/+}$and $D l l 4^{+/ l a c z}$ mice. We focused on VEGFA and VEGF receptor 2 (KDR) in VEGF/KDR pathway, DLL4, JAG1, Notch intracellular domain (NICD), and HES1 in Notch pathway. A recent study identified that Car4 and Apln mark alveolar capillary EC that resemble tip cells, whereas Aplnr mark alveolar capillary EC that resemble stalk cells (20). VEGFA stimulates endothelial angiogenesis and regulates EC survival (21). We noted that $K d r$ expression decreased at P4, whereas Nr2f2 (venous EC marker) and Vegfa expression increased in the whole lung at P4 and P28 in Dll4 ${ }^{+1}$ ${ }_{l a c Z}$ mice (Figure 4A). We also noted increased Nrp1 expression, the Kdr coreceptor in lung EC of Dll4 $4^{+/ l a c Z}$ mice (Figure 4B). We confirmed that VEGFA protein was increased in lungs of Dll4 $^{+/ l a c Z}$ mice by Western blotting (Figure 4, C and D). These data suggest that Dll4 haploinsufficiency programs decreased $K d r$ expression, while inducing compensatory upregulation of Vegfa. Jag1 RNA and protein expression was increased in mouse whole lung (P28) and lung EC (P6, P14) during postnatal lung development (Figure 4, A-D). Expression of NICD, a cleavage product of Notch1, and HES1 was induced strongly in Dll4 ${ }^{+/ l a c Z}$ mouse EC 
A

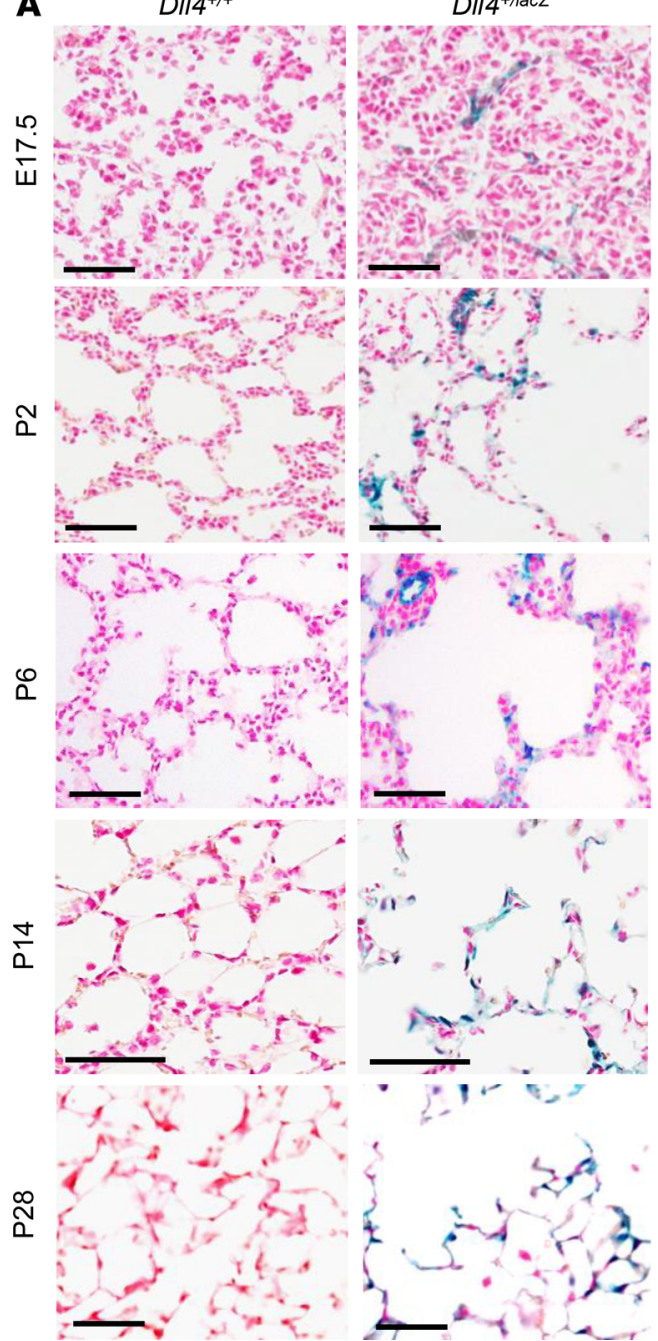

B

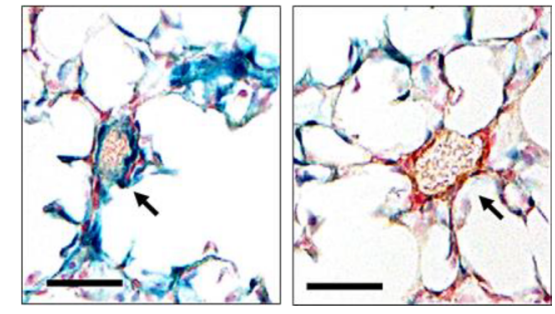

EPHB4 X-gal

C

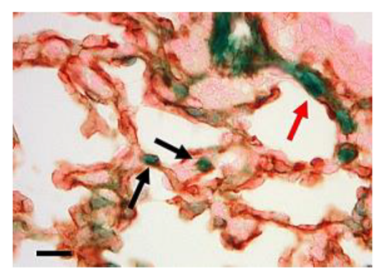

D

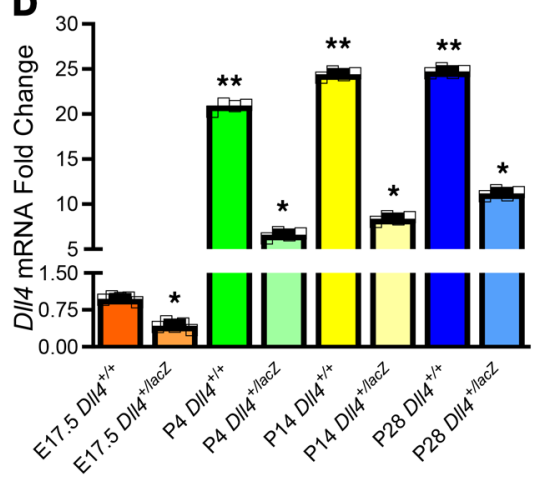

Figure 2. DLL4 lung mapping using the $D I I 4^{+/ / a c z}$ reporter mice. (A) X-gal staining on $D I I 4^{+/+}$and $D I I 4^{+/ l a c z}$ mouse lungs. Blue color represents lacZ gene expression, which indicates DLL4 expression, and fast red (red) represents nucleus ( $n=$ 3). DII4 $4^{+/+}$samples were used as X-gal negative control. (B) X-gal and EPHB4 IHC (brown) staining on P14 mouse sample shows that DLL4 is not expressed in EPHB4+ ${ }^{+}$cells. The arrow (left panel) points to an intermediate Dll4+ EPHB4- blood vessel, and the arrow (right panel) points to a DII4- EPHB4+ blood vessel. (C) X-gal and PECAM IHC (brown) staining on P6 mouse lung sample. The black arrows point to the leading EC of blood vessel, and the red arrow points to a capillary branching from a small artery. (D) qRT-PCR showing mouse whole lung DII4 expression from the canalicular to alveolar stage in WT and DII4 $4^{+/ a c z}$ mice. $n=5$ mice per group; ${ }^{*} P<0.01$, E17.5 DLL4 ${ }^{+/+}$vs. P4, P14, P28 DLL4 ${ }^{+/+}$mice; ${ }^{* *} P<0.01$, E17.5 DLL4+/lacz versus P4, P14, P28 DLL4 ${ }^{+/ l a c z}$ mice. Scale bars: $50 \mu \mathrm{m}$ (A and B); $10 \mu \mathrm{m}$ (C). Data are shown as mean \pm SD throughout. (D) One-way ANOVA with Tukey's test.

(Figure 4, C and D) in relation with increased JAG1 but decreased DLL4. In lung, CAR4 ${ }^{+} / \mathrm{APLN}^{+} \mathrm{EC}$ locate next to AT1 cells and APLNR ${ }^{+}$EC localize to the side away from the air space (20). There was less Car4 and Apln expression and more Aplnr expression in mouse whole lung (P4 and P28) and pulmonary EC (P6 and P14) (Figure 4, A and B). PECAM costaining with CAR4 or APLNR confirmed less CAR4 ${ }^{+}$EC and more ARLPN ${ }^{+}$EC in $D l l 4^{+/ l a c z}$ mouse lung compared with Dll4 $^{+/+}$mouse lung (Figure 4, E and F). We also found that Ephb4 mRNA expression was increased in whole lungs at P4 and P28,and that Efnb2 mRNA expression was decreased at P6 but increased at P28 in pulmonary EC (Figure 4, A and B). These data indicate polarization of lung EC toward a JAG1/NICD/HES1 fate in Dll4 ${ }^{+/ l a c z}$ mice, and the lung capillary EC population shifts to an $\mathrm{Aplnr}^{+}$expression signature from a $\mathrm{Car}^{+} / \mathrm{Apln}^{+}$signature.

Dll4haploinsufficiencycausesdefective vascularnetworkin the developinglung. Toinvestigate the effect of Dll4haploinsufficiency on lung vascular arborization, we performed PECAM mapping. PECAM whole-mount lung staining at E14.5 revealed subtle increase in vascular loops in lll4 $^{+/ l a c Z}$ mice with denser networks (Figure 5A). 

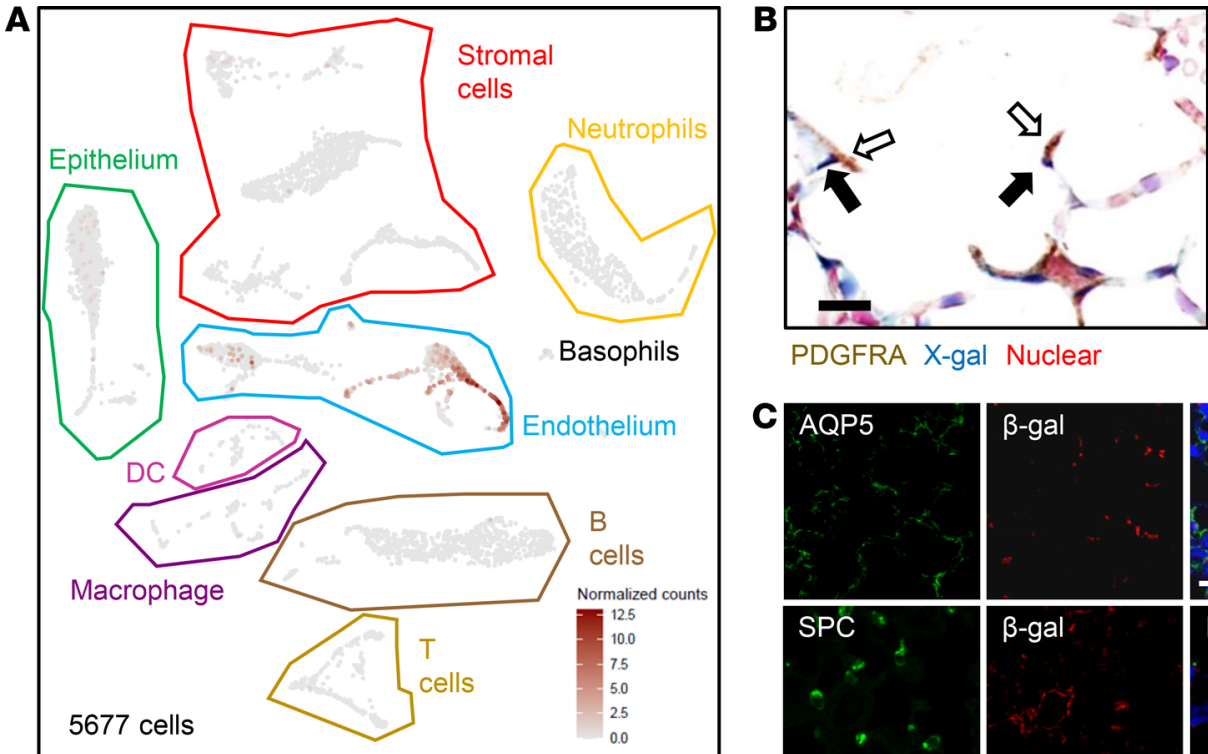

PDGFRA X-gal Nuclear
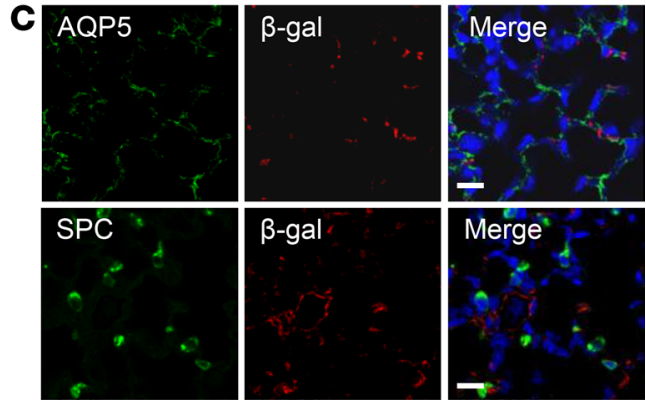

Figure 3. DLL4 prominently expresses in EC. (A) Three C57BL/6 WT mouse lungs were collected on P14 for single-cell RNA-Seq, and DII4 expression plot was generated from 5677 cells. Epithelium, endothelium (EC), and stromal cells were bordered by green, blue, and red lines separately. Note: DLL4 (brown dots) expression is predominantly in the EC cluster. (B) DII4+/lacz mouse lungs were harvested and cryosections were obtained on P14, and then X-gal staining and PDCFRA IHC staining were performed. Blue, brown, and red colors indicate X-gal, PDGFRA, and nuclear staining, respectively. Hollow arrows point to PDGFRA ${ }^{+}$cells, and solid arrows point to X-gal ${ }^{+}$cells. (c) Mouse lungs were harvested from 14-day-old DIl/4/lacz mice. AQP5 (green) and SPC (green) (IF) were costained with $\beta$-galactosidase (red) separately. $n=5$ per group. Scale bars: $10 \mu \mathrm{m}$.

Additionally, we noted exuberant, misaligned capillary growth in $D l l 4^{+/ l a c Z}$ mice with fewer bronchioles at E17.5 (Figure 5B). At P6, a double capillary network was visualized in $D_{l l 4^{+/+}}$mice; however, in Dll4 $^{+/ l a c z}$ mice we found "whorls" of misaligned, dysmorphic capillary networks (Figure 5C). We posited that deviant capillary arborization will impair microvascularization in the alveolar phase. At P14, the number of blood vessels ranging in size 10-150 $\mu \mathrm{m}$ decreased by $50 \%$ (Figure $5, \mathrm{D}$ and $\mathrm{E}$ ), whereas the average capillary diameter reduced from 3.8 to $2.7 \mu \mathrm{m}(P<0.001)$ in $D l l 4^{+/ l a c z}$ mice (Figure $5, \mathrm{~F}$ and $\left.\mathrm{G}\right)$. To verify whether Dll4 haploinsufficiency influences EC proliferation, Ki67 and ERG double IF staining was performed on P14 mouse lung sections, which revealed more EC proliferation in Dll4 ${ }^{+/ l a c Z}$ lung EC (Figure 5, H and I). However, we did not observe significant differences in lung EC apoptosis with ERG and TUNNEL double staining between $D_{l l 4^{+/+}}$and Dll4 ${ }^{+/ L a c Z}$ mice at P14 (data not shown). These data demonstrate that Dll4 heterozygosity stimulates EC proliferation and programs dysmorphic vascular patterning characterized by hypervascular, misaligned vessels in the canalicular and saccular stages, and decreased capillary caliber and number of small pulmonary blood vessels in the alveolar stage.

Dysmorphic vascular development in Dll4 ${ }^{+/ l a c Z}$ mice impairs alveolarization. DLL4/PECAM IF staining revealed DLL4 is expressed in the leading EC of capillaries in alveolar septa of human and mouse lungs (Figure 6A), with X-gal/PECAM IHC staining confirming DLL4 in the alveolar septum of Dll4 ${ }^{+/ l a c Z}$ mice (Figure 6B). We posited that deviant vascular patterning seen with Dll4 haploinsufficiency will impact alveolar septal development and alveolarization. As early as the canalicular stage, we noted that terminal bronchiole development was impaired in $D l l 4^{+/ l a c Z}$ mice (Figure 5B). Blunt primary and underdeveloped secondary septa were observed at P6 in Dll4 ${ }^{+/ l a c Z}$ mouse lungs (Figure 6C). Lung morphology was clearly altered by the saccular stage (P2) (Figure 6D), and lung morphometry during the early and mid-alveolar phase (P8 and P14) as well as in the mature lung (P28) revealed decreased radial alveolar counts and increased mean linear intercepts in Dll4 ${ }^{+/ l a c Z}$ mice compared with Dll4 ${ }^{+/+}$littermates (Figure 6, D-F). Impaired alveolarization in Dll4 ${ }^{+/ l a c Z}$ mice was observed in both male and female pups. Although it is not technically feasible to inflate P2 lungs, and alveoli are not formed, we did assess the number of completed air sacs distal to the respiratory bronchioles. We found a $25 \%$ reduction in the number of distal air spaces (Dll4 ${ }^{+/ l a c Z}$ vs. Dll4 ${ }^{+/+} ; 1.7 \pm 0.5$ vs. $2.4 \pm 0.4 \mathrm{SD}, P<0.02, n=9$ and 5 , respectively). These data demonstrate that DLL4 is required for lung alveolarization, and loss of 1 Dll4 copy disrupts distal lung morphogenesis. 
A

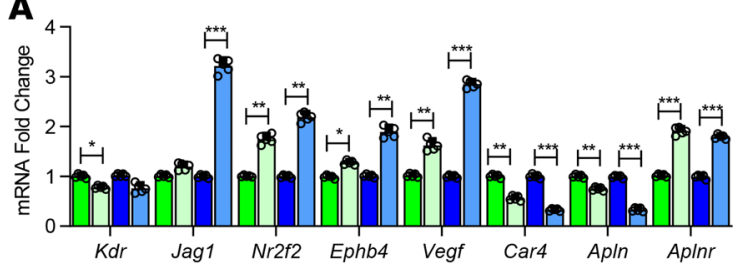

口 P4 DIII $4^{+/+}$口 P4 DIII $4^{+/ l a c Z}$

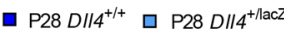

B

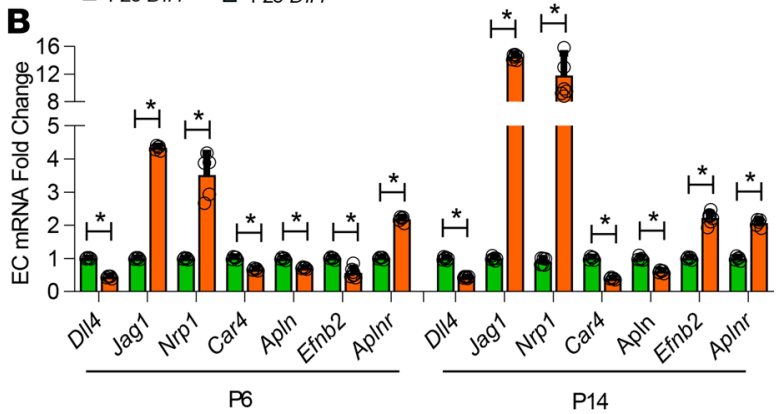

D $/ / 4^{+/+} \square D / 14^{+/ l a c Z}$

C

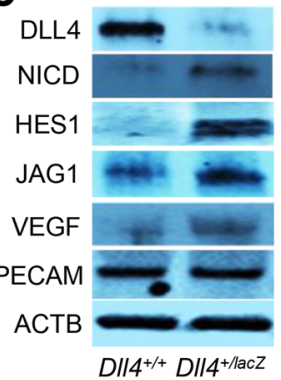

D

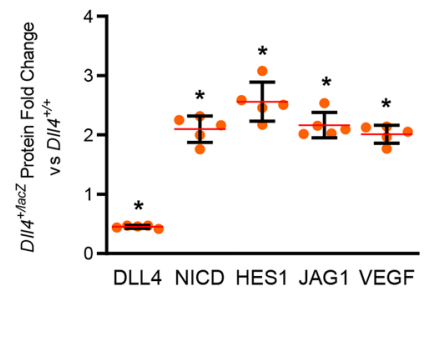

E
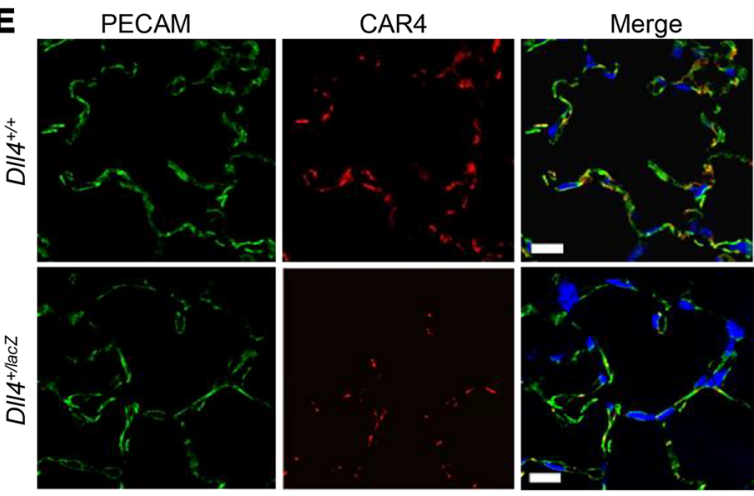

$\mathbf{F}$
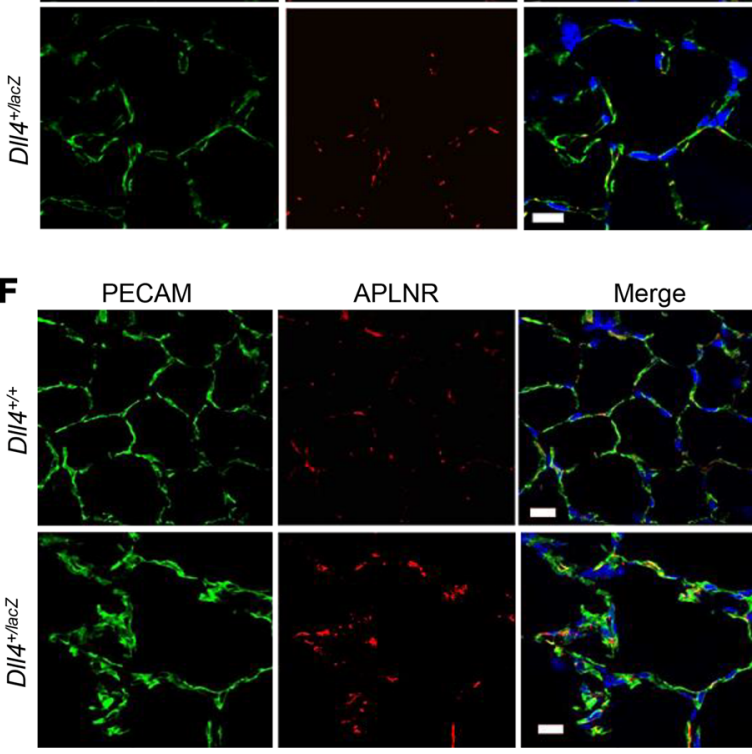

Figure 4. Altered EC gene expression in the ECs of DII4+/lacz mice. (A) Whole-lung RNA homogenates obtained from DII4 ${ }^{+/+}$and DII4 $4^{+/ l a c z}$ at P4 and P28 were used to quantify $K d r$, Jag1, Nr2f2, Ephb4, Vegfa, Car4, Esm1, Apln, and Aplnr with qRT-PCR. $n=5$ mice per group; ${ }^{*} P<0.05$, ${ }^{*} P<$ 0.01 , ${ }^{* *} P<0.001$. (B) Mouse lung ECs (PECAM pulldown) obtained from $\mathrm{DI} / 4^{+/+}$and $\mathrm{D} / 14^{+/ l a c z}$ at P6 and P14 were used to quantify DII4, Nrp1, Jag1, Car4, Apln, Esm1, Efnb2, and Aplnr. n = 5 mice per group; ${ }^{*} P<0.01$. (C) Mouse lung ECs were harvested from 14-day-old $\mathrm{DI} / 4^{+/+}$and DI/4+/lacz mice. Lung homogenates were used to quantify DLL4, NICD, HES1, JAG1, VEGFA, and PECAM by immunoblotting, with densitometry shown graphically (D). $n=5$ mice per group; ${ }^{*} P<0.01$. (E) PECAM (green), CAR4 (red), and DAPI staining on P14 DI/4 ${ }^{+/+}$and DI/4+/lacz lung sections $(n=4)$. (F) PECAM (green), APLNR (red) and DAPI (blue) staining on DII4+/+ and DI/4+/lacz mouse lung slides $(n=4)$. Scale bars: 10 $\mu \mathrm{m}$. Data are shown as mean $\pm \mathrm{SD}$. (A, B, and $\mathbf{D}$, Gaussian distribution used 1-way ANOVA with Tukey's test) (A and B) Non-Gaussian distribution used 2-tailed Mann-Whitney $U$ test. 
A

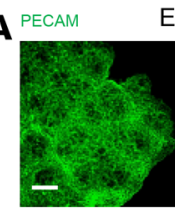

$D / 14^{+/+}$
E14.5

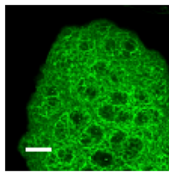

DII4+/lacZ
C

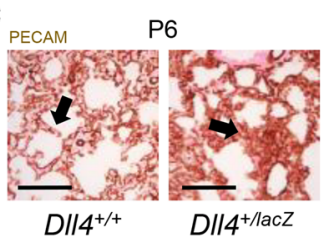

E

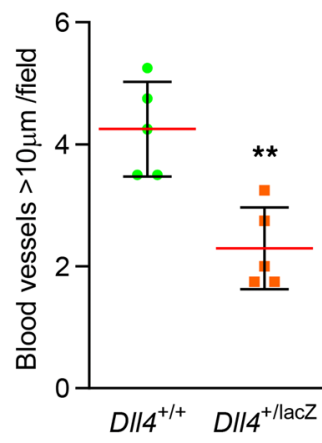

H

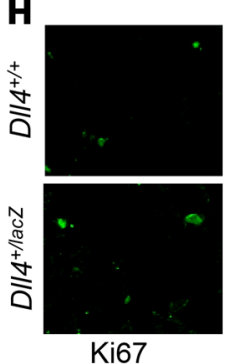

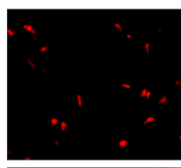

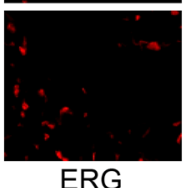

B $\mathrm{PE}$

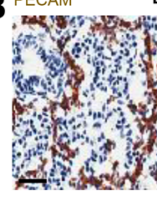

$D / 14^{+/}$
E17.5

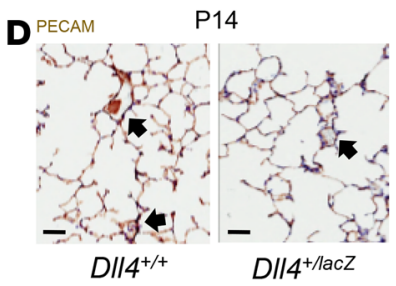

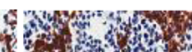

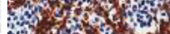

D/14 $+/ / a c Z$

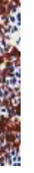

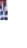

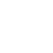


A
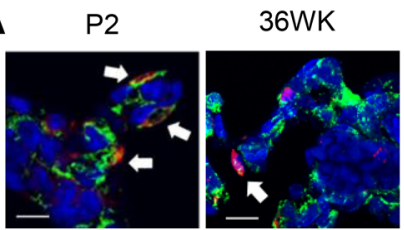

B

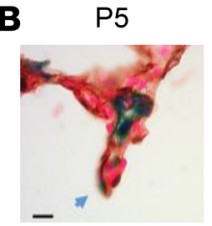

C

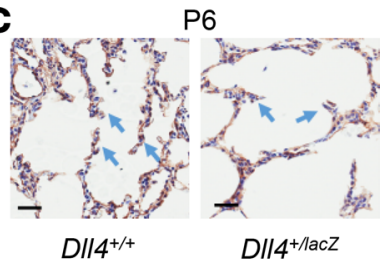

D $\quad$ P2

D 2

P8

$\mathrm{P} 14$

$\mathrm{P} 28$
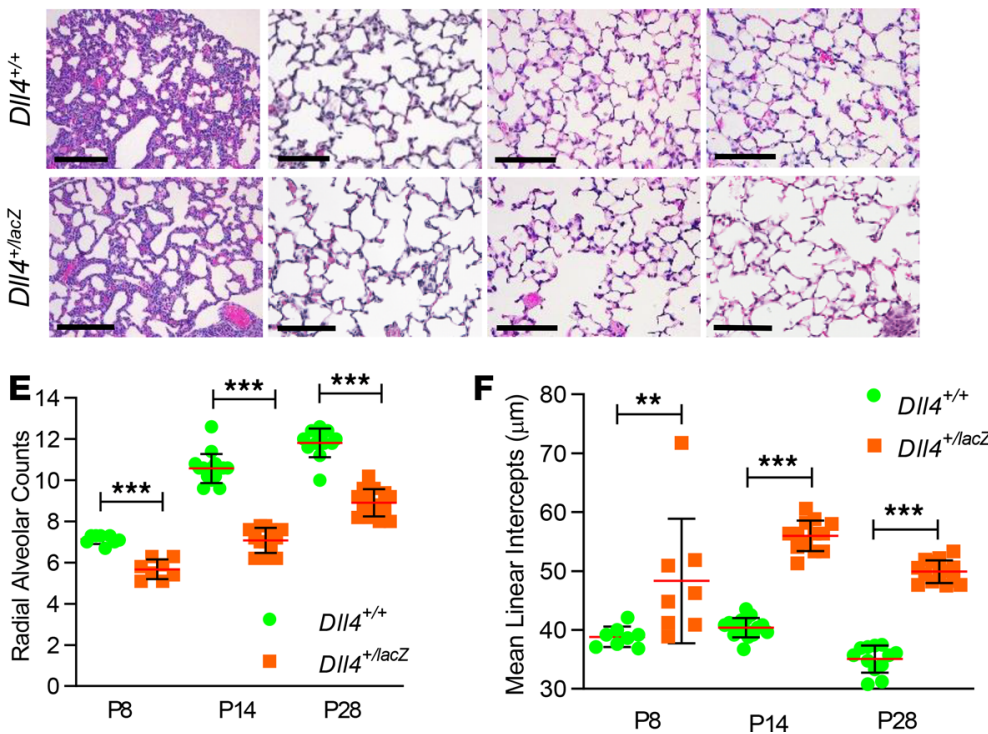

Figure 6. DII4 haploinsufficiency causes defective alveolarization. (A) DLL4 (red), PECAM (green), and DAPI (blue) staining indicates DLL4 is expressed in the leading EC localized in primary septa at P2 in mouse lung (left panel) and at 36 weeks in human lung (right panel). The arrows point to the DLL4+ leading EC. (B) X-gal (blue) and PECAM IHC (brown) staining shows that DLL4 is expressed in the leading EC localized in primary septa at P6 in mouse lung. The arrow points to the DLL4+ leading

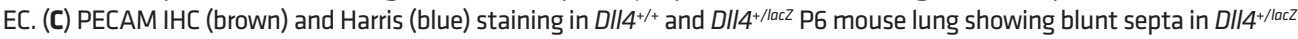
compared with $\mathrm{DII} 4^{+/+}$. The arrows point to normal septa in $\mathrm{DII} 4^{+/+}$in the left panel and blunt septa in $\mathrm{DII} 4^{+/ / a c z}$ in the right panel. (D) H\&E staining on P2, P8, P14, and P28 DII4+/+ and DII4//lacz mouse lungs. Radial alveolar counts (RAC) (E) and mean linear intercepts (MLI) (F) at P8, P14, and P28 shown graphically. $n=5$ mice/group, ${ }^{* *} P<0.01$, ${ }^{* *} P<0.005$. Scale bars: $10 \mu \mathrm{m}$ ( $\mathbf{A}$ and B); $25 \mu \mathrm{m}$ (C); $100 \mu \mathrm{m}$ (E). Data are shown as mean \pm SD. (F) Two-tailed Mann-Whitney $U$ test.

implicated in pathological angiogenesis were upregulated, whereas genes known to suppress angiogenesis such as Stat $1(\mathrm{FC}=0.59, P=0.02), \operatorname{Il1} 7 \mathrm{~F}(\mathrm{FC}=0.12, P=0.049)$, and $K l f 2(\mathrm{FC}=0.74, P=0.02)$ were suppressed. We then performed exploratory analysis to determine whether the lack of Dll4 altered maturation of alveolar type I (AT1) or alveolar type II (AT2) cells, key constituents of the mature alveoli. Ingenuity pathway analysis predicted delayed maturation of AT1 cells in Dll4 ${ }^{+/ l a c Z}$ mice based on decreased Esr2 (FC $\left.=0.32, P<10^{-4}\right)$ and $K l f 2(\mathrm{FC}=0.74, P<0.02)$ expression. Validation of RNA-Seq data by quantitative reverse transcription PCR (qRT-PCR) in independent lung samples from $\mathrm{Dll}^{+/+}$and $\mathrm{Dll} 4^{+/ l a c z}$ mice confirmed progressive decrease in Esr2 and Klf2 expression on P4, P14, and P28 with Dll4 deficiency (Figure 7B). We next quantified temporal changes in expression of AT1 lineage markers Hopx, Aqpn5, and Pdpn in $\mathrm{Dll}^{+/ l a c Z}$ and $\mathrm{Dll}^{+/+}$littermates. Hopx expression was decreased on P4, but all AT1 markers were decreased on P14 and P28 in Dll4 ${ }^{+/ a c z}$ mice (Figure 7B). AQP5 ${ }^{+}$AT1 cells were continuously distributed along alveolar inner lining surface in $\mathrm{Dll}^{+/+}$lungs, but showed an interrupted pattern in $\mathrm{Dll}^{+/ l a c z}$ lungs (Figure 7C). $\mathrm{SPC}^{+}$AT2 cells were increased in Dll4 ${ }^{+/ l a c z}$ lungs at P15 (Figure 7, C and D). Immunoblotting and PCR confirmed there was more SPC expression in developing lungs at P14 and P28 (Figure 7, B, E, and F). PDGFRA $^{+}$fibroblasts interact with EC and alveolar epithelial cells, and secrete extracellular matrix molecules in the newly formed septa during lung alveolarization (22), and, therefore, we quantified PDGFRA expression and PDGFRA ${ }^{+}$cells. We found increased PDGFRA RNA and protein expression at P14 and P28 (Figure 7, B, E, and F) and more PDGFRA ${ }^{+}$cells at P14 with IHC staining (Figure 7, G and H) in Dll4 ${ }^{+/ l a c z}$ lungs. These data imply Dll4 deficiency programs aberrant AT1 development with increases in AT2 and PDGFRA ${ }^{+}$fibroblasts that contribute to impaired lung alveolarization. 
A

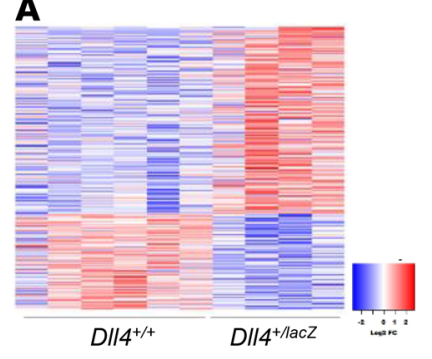

C
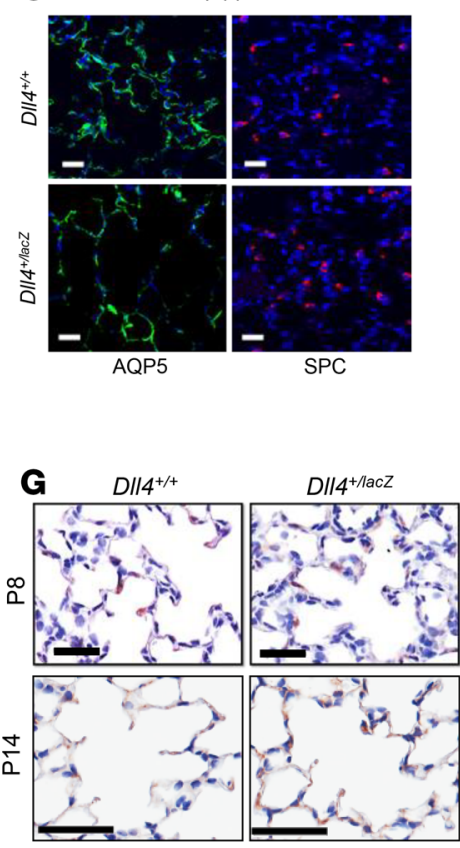

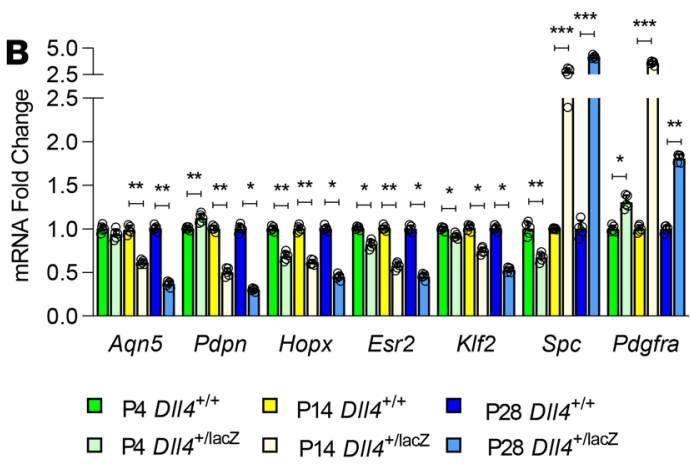

D

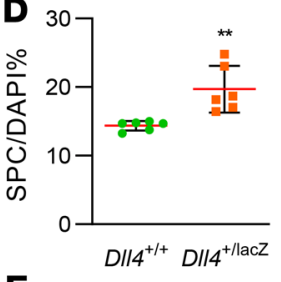

E
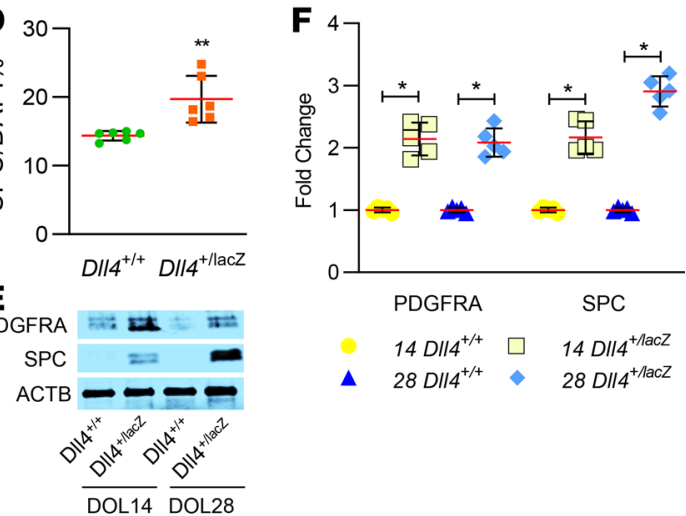

Figure 7. RNA-Seq identifies lung genes associated with deviant vascular and alveolar development with

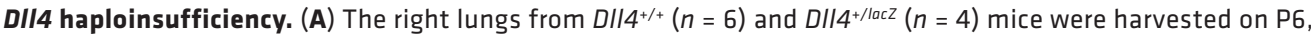
and RNA extracted was used for RNA-Seq using an Illumina NovaSeq platform. Heatmap of 336 differentially expressed genes between DI/4+/+ $(n=4)$ and DII4+/lacz $(n=4)$ mouse lungs $(P<0.05)$. (B) qRT-PCR demonstrates Aqp5, Pdpn, Hopx, Esr2, KIf2, Spc, and Pdgfro expression in DI/4+/+ and DII4+/lacz mouse lungs at P4, P14, and P28. (C) AQP5 (green) and SPC (red) IF staining with DAPI (blue) in D/14 ${ }^{+/+}$and D $1 / 4^{+/ l a c z}$ mouse lungs at P14, with quantification shown for percentage of SPC ${ }^{+}$cells of the total DAPI-stained cells (D), $n=6$ in each group and $P<0.005$. (E) Mouse lungs were harvested from D/14+/+ and D/14+/lacZ mice at P14 and P28. Lung homogenates were used to quantify SPC and PDGFRA by immunoblotting, with densitometry shown graphically (F), $n=5$ mice per group; $P<0.01$. (G) PDGFRA (brown) IHC staining with Harris staining (blue) on P8 and P14 DI/4 $4^{+/+}$and $\mathrm{D} / 14^{+/ l a c z}$ mouse lung sections, with quantification shown for percentage of PDGFRA ${ }^{+}$cells in total Harris-stained cells $(\mathbf{H}), n=6$ in each group, ${ }^{*} P<0.05,{ }^{*} P<0.01$, and ${ }^{* * *} P<0.001$. Scale bar: $20 \mu \mathrm{m}$. Data are shown as mean \pm SD. (B, F, and H) Gaussian distribution used 1-way ANOVA with Tukey's test. (D) Gaussian distribution with 1 comparison used 2-tailed $t$ test with Welch's correction.

Dll4 deficiency causes deviant angiogenesis in human lung ECS. We next investigated whether DLL4 deficiency programs deviant angiogenesis in human lung ECs (HLECs). As primary HLECs do not survive 3D-angiogenesis assays, we immortalized primary HLEC (HLEC-Im) SV40 transformation. We then knocked down DLL4 expression using lentivirus containing DLL4 shRNA and mCherry in HLEC-Im. EC phenotype of HLEC-Im was confirmed with ERG and CDH5 staining, and AcLDL uptake assay (Supplemental Figure 1A). We sorted cells into 4 groups based on mCherry intensity (Supplemental Figure 1B), and then verified that medium mCherry intensity cells had about 50\% Dll4 knockdown (Figure 8B). 3D angiogenesis assays demonstrated that DLL4 deficiency caused more EC proliferation, hyberbranching, dysmorphic and nondirectional angiogenesis in HLEC-Im (Figure 8, A and C). 
To confirm 3D angiogenesis data in primary fetal HLEC, we used DLL4 siRNA to reduce DLL4 expression by $50 \%$ (Figure 8E). DLL4-deficient HLEC had increased tube and network formation in 2D Matrigel assays (Figure 8, D and F). In Dll4 ${ }^{+/ l a c Z}$ mice, we had noted upregulation of JAG1-NICD signaling with DLL4 deficiency. To determine this signature shift is relevant in HLEC, and is cell autonomous, we quantified $J A G 1$ and HES1 mRNA expression and found their expression increased with DLL4 deficiency (Figure 8G). Silencing DLL4 induced protein expression of JAG1, HES1, and NICD in parallel with increased expression of the venous marker, NR2F2 (Figure $8, \mathrm{H}$ and I). To study whether upregulated NICD in DLL4-deficient EC is induced by increased JAG1, we knocked down DLL4 and JAG1 with siRNA separately and simultaneously. NICD expression was increased in DLL4-deficient EC, but decreased in $J A G 1$-deficient EC (Figure 8, J and K). Interestingly, NICD expression was repressed in DLL4 and JAG1 double knockdown EC (Figure 8, J and K). These data confirm that DLL4 deficiency cell autonomously induces JAG1-NICD signaling and deviant hypersprouting angiogenesis phenotype in human EC.

Hyperoxia represses VEGF and DLL4 expression in the mouse lung and HLEC. To investigate the relevance of DLL4 in neonatal lung disease, we used a neonatal model of hyperoxic lung injury in vivo and in vitro. We observed that VEGF and DLL4 protein and RNA expression in mouse lung was repressed by 3 days of $85 \% \mathrm{O}_{2}$ treatment from P3 to P6 (Supplemental Figure 2, A-C). We also confirmed that VEGF and DLL4 protein expression in primary HLEC was repressed by 2 days of $85 \% \mathrm{O}_{2}$ treatment (Supplemental Figure 2, D and E). These data showed that hyperoxia, a major risk factor for BPD, reduces DLL4 and VEGF expression in HLEC and mouse lungs.

\section{Discussion}

The functional role of sprouting angiogenesis in distal lung microvascular arborization and alveolarization remains uncharacterized $(1,8,9)$. Using mapping strategies, we show that DLL4 is expressed in both human and mouse lung from the canalicular through the alveolar stage of lung development. Dll4 haploinsufficiency results in deviant lung microvascularization with an early "hypersprouting" angiogenic phenotype, followed by smaller capillaries and fewer distal vessels. These phenotypic changes occurred in parallel with skewing of lung EC expression signature toward JAG1/NICD activation and venous EC specification. Induced $D L L 4$ deficiency in human fetal lung EC autonomously programmed exaggerated angiogenic sprouting in vitro, reproducing an exaggerated JAG1/NICD activation signature. Strikingly, we noted that Dll4 deficiency impaired distal lung acinar development from the canalicular stage with suppression of AT1 lineage markers (Figure 9). These data reveal an obligate role for DLL4 in pulmonary microvascular growth, arborization, and alveolarization.

Mapping strategies in $\mathrm{C} 57 \mathrm{BL} / 6, \mathrm{Dll}^{+/ l a c Z}$ reporter mice, and human autopsy samples revealed signatures of DLL4 expression from the canalicular stage, with exponential increases from the saccular through alveolar stage. DLL4 expression was found in EC lining of lung arteries and in capillaries, but not veins, mimicking embryonal patterns $(12,23)$. DLL4 expression did not colocalize with AT1, AT2, or PDGFRA ${ }^{+}$ cells. scRNA-Seq confirmed that Dll4 is predominantly expressed in the lung EC cluster on P14, consistent with similar distribution patterns during other stages of lung development in LungMap. We found DLL4 in the leading cells of $\mathrm{PECAM}^{+}$staining capillaries, indicating sprouting angiogenesis $(24,25)$. Interestingly, temporal increase in DLL4 expression in the saccular and alveolar phase coincides with the rapid increase in lung microvascular growth postnatally. These mapping studies suggest a role for DLL4-regulated angiogenesis during distal lung ontogeny. Prior work by DeMello et al. based on morphological analysis of lung vascular casts suggested that vasculogenesis was critical for distal lung vascular development (6). Parera et al. mapped TIE2 (an EC receptor for angiopoietins) to suggest that angiogenesis contributes to distal lung vascularization (8). Use of lung vascular casts or mapping the ubiquitous EC receptor, TIE2, did not clarify the molecular mechanisms regulating distal lung vascularization, nor functionally examine the contribution of angiogenesis to alveolarization. Lung stage-specific mapping combined with morphological analysis clarifies the role of DLL4 in lung development.

$D_{l l 4^{-/}}$and Dll4 ${ }^{+/-}$mice on the C57BL/6 background do not survive to the canalicular stage, dying by E11.5 of severe vascular defects. We therefore used $D l l 4^{+/ l a c z}$ mice on the CD1 background to study the impact of Dll4 deficiency on lung development. We found that Dll4 haploinsufficiency altered lung EC tip versus stalk cell behavior programming a hypersprouting and nondirectional capillary arborization pattern evident from the canalicular phase. This dysmorphic lung vascular patterning is similar to the hypersprouting, nonproductive angiogenesis phenotype described with Dll4 deficiency during retinal vascularization $(23,26)$. 
A

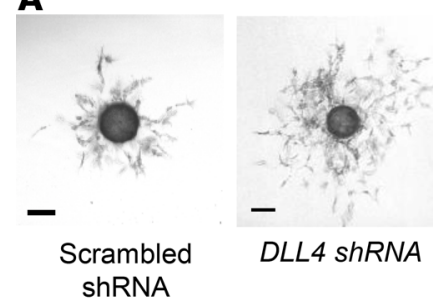

D

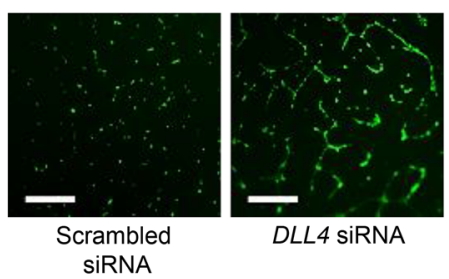

E

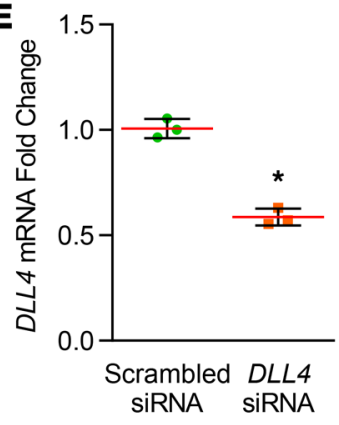

C
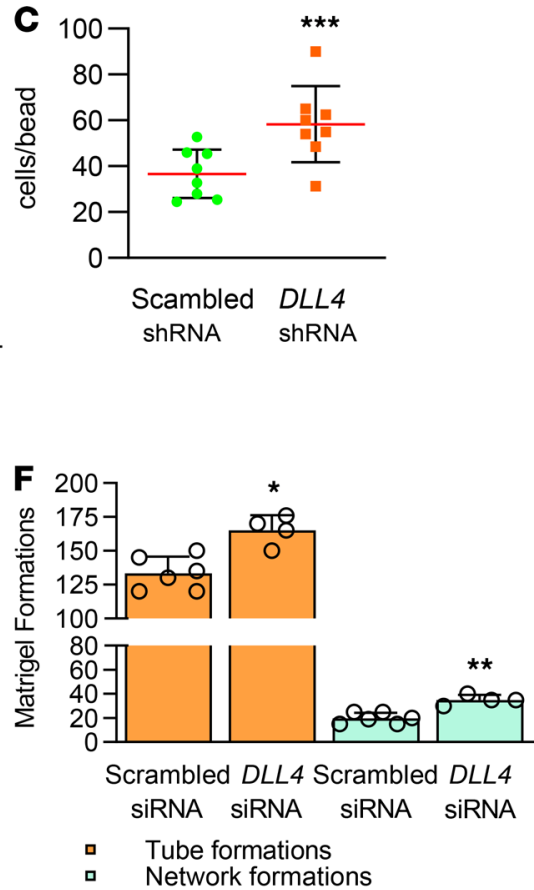

I

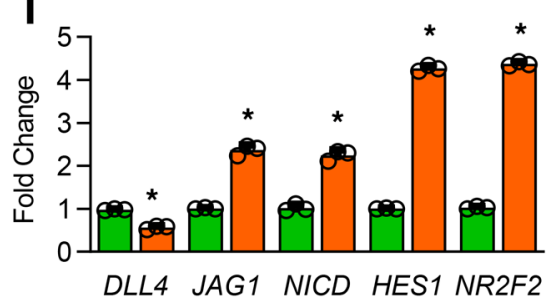

- Control

- Control + siDLL4

- $\quad$ Scrambled siRNA

- DLL4 siRNa
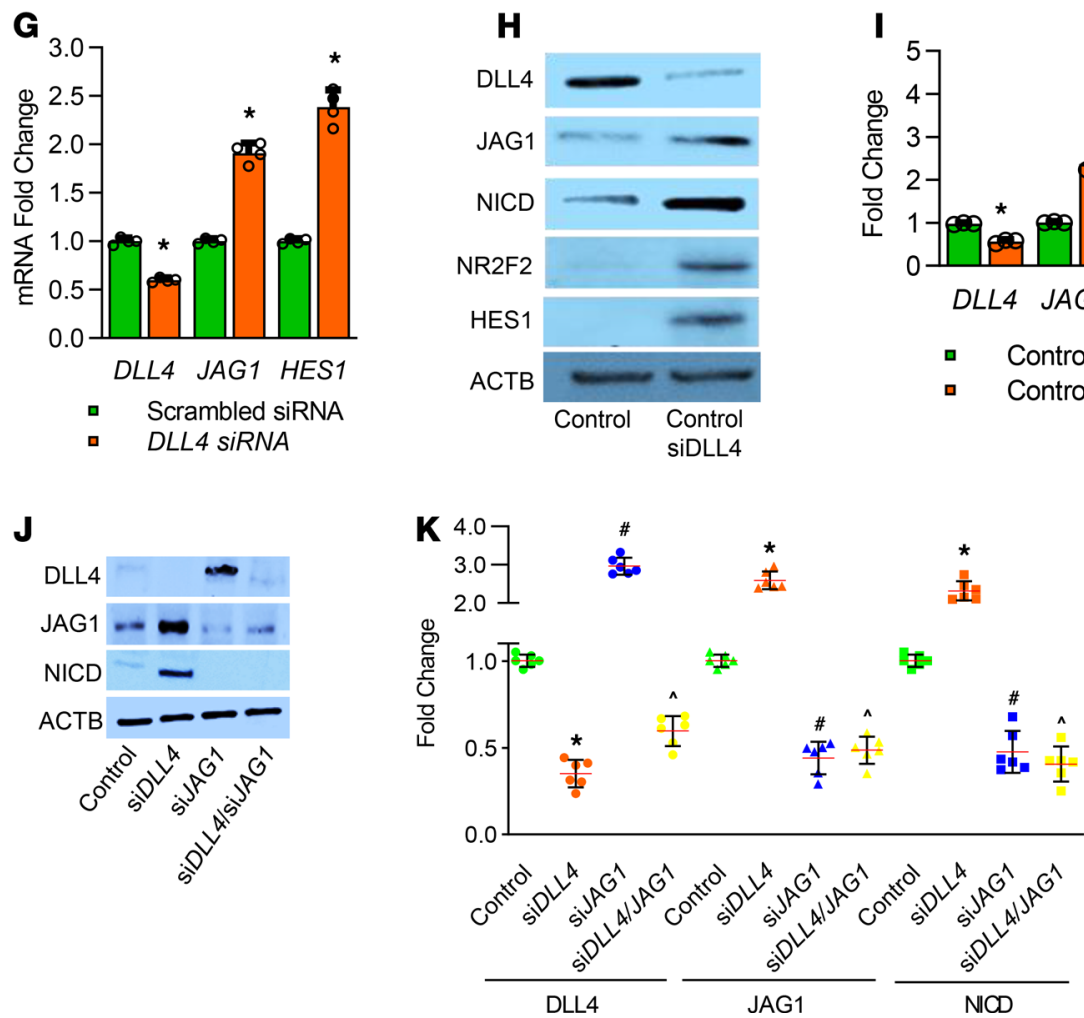

Figure 8. DLL4 deficiency programs a hypersprouting angiogenic phenotype in HLEC in vitro. (A) DLL4-deficient HLEC-Im show hyperbranching network in 3D cell culture compared with control EC, with quantifications shown for average cell numbers per bead (C), 1-4 beads per well, 8 wells in both groups, $P<0.001$. (B) qRT-PCR indicates that DII4 is knocked down around 50\% in DLL4-deficient HLEC-Im. $n=3$ per group, $P<0.01$. (D) DLL4 partially silenced in primary HLEC using siDLL4 shows increased branching and network formation in Matrigel compared with EC treated with scrambled siRNA with quantifications shown graphically (F), ${ }^{*} P<0.02$, ${ }^{* *} P<0.01$. (E) qRT-PCR indicates that siDLL4 reduced $D L L 4$ in primary HLEC by around $50 \% . N=4$ per group, $P<0.03$. (C) qRT-PCR from HLEC shows a $50 \%$ decrease with siDLL4 and an increase in both JAG1 and HES1. $n=4$ per group; ${ }^{*} P<0.03$, control vs. siDLL4. (H) HLEC were harvested after siRNA treatment and homogenates were used to quantify DLL4, JAG1, NICD, NR2F2, and HES1 by immunoblotting, with densitometry shown graphically (I). $n=3$ per group; ${ }^{*} P<$ 0.01 , scrambled siRNA vs. siDLL4. (J) HLEC were harvested after scrambled siRNA, siDLL4 or si/AC1 treatment and homogenates were used to quantify DLL4, JAG1, and NICD by immunoblotting, with densitometry shown graphically (K). $n=5$ per group; ${ }^{*} P<0.01$, control vs. siDLL4; ${ }^{\circ} P<0.01$, control vs. siJAG1; ${ }^{\wedge} P<0.01$, control vs. siDLL4/siJAG1. Scale bars: $250 \mu \mathrm{m}$ (A); $100 \mu \mathrm{m}$ (C). Data are shown as mean \pm SD. (C and K) Gaussian distribution used 1-way ANOVA with Tukey's test) (B, E, F, and I) Gaussian distribution with 1 comparison used 2-tailed $t$ test with Welch's correction. (C) Non-Gaussian distribution used 2-tailed Mann-Whitney $U$ test. 


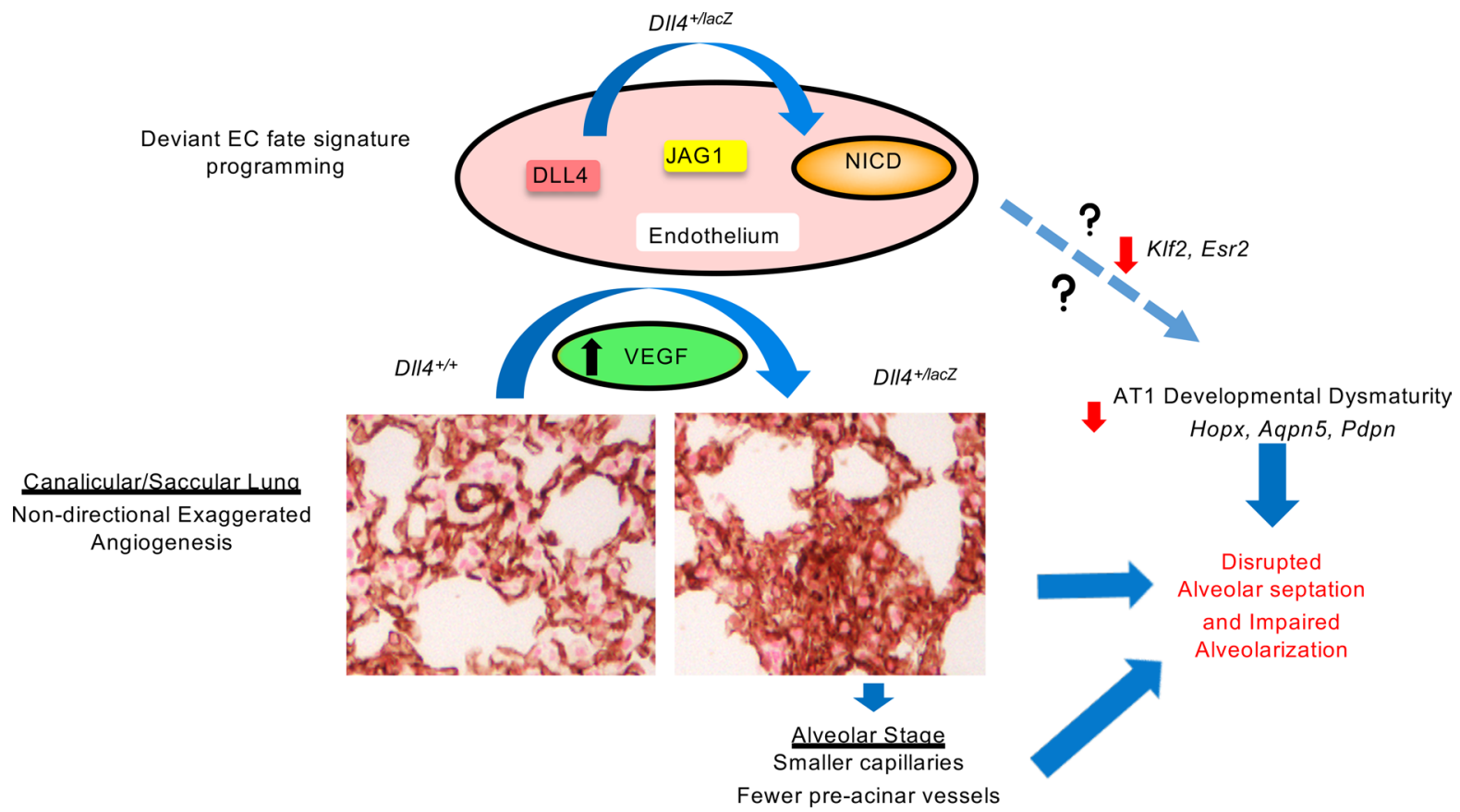

Figure 9. Illustration demonstrating mechanisms by which DLL4 haploinsufficiency programs altered lung vascular and alveolar development in mice. AQP5, aquaporin 5; AT1, alveolar type I cell; DLL4, delta-like 4; Esr2, estrogen receptor 2; HOPX, homeodomain-only protein homeobox; JAG1, Jagged 1; KIf2, Kruppel-like factor 2; NICD, Notch intracytoplasmic domain; PDPN, podoplanin; VEGF, vascular endothelial growth factor.

In the retina, DLL4 represses tip cell division in cis and in trans specifies a stalk cell phenotype. Dll4 heterozygosity induces more tip cells and sprouting angiogenesis in the leading retinal edge, and less regression in the central retina $(13,27)$. Increased Ki67 staining in lung EC of Dll4 ${ }^{+/ l a c z}$ mice further supports the proliferative, nondirectional nature of the EC angiogenic behavior. As lung development progressed, the earlier dysmorphic arborization progressed to decreased capillary thickness and fewer intermediate (10-150 $\mu \mathrm{M})$ size blood vessels, suggesting that the earlier "nonproductive" angiogenesis phenotype disrupts microvascular growth during alveolarization. These data evince that DLL4-regulated angiogenesis is required for microvascular growth and arborization from the canalicular stage of lung development.

We examined the mechanisms by which Dll4 deficiency programs dysmorphic vascular development. Dll4 heterozygosity induced lung EC Vegfa and Nrp1 expression. Increased VEGFA expression in Dll4 ${ }^{+/ L a c Z}$ mice could be a regulatory autocrine mechanism related to decreased DLL4 as described in the retina (13). Whether the combination of increased Vegfa and decreased Dll4 in Dll4 ${ }^{+/ l a c Z}$ EC dysregulates VEGFA-DLL4 gradient-specified EC tip cell migration during sprouting angiogenesis needs to be studied. Previous studies have shown that increased NRP1 in the presence of increased VEGFA induces angiogenesis (28). Our results showing exaggerated angiogenesis in Dll4 ${ }^{+/ l a c Z}$ mice in the presence of increased Vegfa and Nrpl is consistent with the above. Interestingly, we also found that Jag1 was strongly induced in mouse lung EC of Dll4 $4^{+/ l a c Z}$ mice. Dll4 and Jag1 serve opposing functional roles in regulating angiogenesis in retina, although both of them are Notch1 ligands (29). Dll4 represses angiogenesis, whereas Jag1 stimulates angiogenesis in the retina. EC Jag1 deficiency disrupts vascularization, whereas Jag1 overexpression induces exaggerated angiogenesis in the retina (29). Our lung data showing exaggerated angiogenesis in association with increased EC JAG1 in Dll4 ${ }^{+/ l a c z}$ mice are consistent with retinal data. Increased NICD expression in Dll4 ${ }^{+/ l a c z}$ lung EC may be driven by JAG1, and potentially induces a different EC transcriptional program compared with DLL4-NICD signaling. In summary, our data show that lack of Dll4 induces Jag1, Nrp1, and NICD, which in the presence of increased Vegfa programs aberrant hypersprouting angiogenic phenotype. In contrast to the retina, a recent study by Vila Elis et al. suggested that traditional markers of EC tip versus stalk cell specification cannot be defined in the alveolar niche $(13,20,23)$. Alveolar capillary EC expressing Car4 under AT1 VEGF stimulation (Plvap ${ }^{-}, A_{p l n^{+}}$) exhibit tip cell-like behavior, whereas alveolar capillary EC that do not express $\operatorname{Car} 4\left(\right.$ Plvap $\left.^{+}, A p l n r^{+}\right)$exhibit more stalk cell-like behavior. Our data show that 
D114 haploinsufficiency decreases EC expression of Car4 and Apln, whereas Aplnr expression increases, suggesting a polarization toward stalk cell-like behavior. Studies examining the relationship between VEGF, CAR4, and DLL4 in regulating angiogenesis, EC specification, and AT1 interactions will be pursued in the future.

To identify novel, lung-specific mechanisms that could program deviant vascularization in Dll4 ${ }^{+/ l a c z}$ mice we performed RNA-Seq. Genes reported to regulate pathological angiogenic signaling increased, whereas genes that suppress angiogenesis were decreased in Dll4 $4^{+/ l a c Z}$ mice. Foxn 4 and Thbs 4 are proangiogenic by regulating Notch and EC migration $(30,31)$, whereas $C c l 2$ and $\operatorname{Lrg} 1$ are NF- $\mathrm{kB}$-regulated genes implicated in inflammatory angiogenesis $(32,33)$. Stat1, Klf2, and Il17f expression, which suppress angiogenesis in pathological conditions, was inhibited (34-37). Whether these pathways are specific to the lung or mediate deviant angiogenesis phenotypes described in the retina or embryogenesis with DLL4 deficiency remains unknown. Although the mechanistic relationships between DLL4 and novel targets identified to the overall vascular phenotype in Dll4 $4^{+/ l a c Z}$ mice need to be studied, these data suggest that Dll4 haploinsufficiency programs a dysregulated angiogenic phenotype in the lung.

To investigate whether the effect of Dll4 deficiency on aberrant angiogenesis is cell autonomous and is relevant to human primary lung EC, we pursued studies in HLEC. We immortalized primary HLEC to HLEC-Im to investigate the effect of permanent D114 deficiency on angiogenic behavior in robust 3D-angiogenesis assays. HLEC-Im with 50\% DLL4 knockdown demonstrated exuberant endothelial sprouting, with disorganized network growth and branching, consistent with in vivo phenomenon noted in Dll4 ${ }^{+/ l a c Z}$ mouse lung EC. Studies in primary fetal HLEC using transient siRNA-based knockdown also revealed similar effects with exaggerated branching and network formation with partial D114 deficiency. We also noted that transient $D L L 4$ silencing upregulated JAG1, NICD, and HES1 in primary HLEC, consistent with our in vivo data in $D l l 4^{+/ l a c Z}$ mice. Interestingly, silencing JAG1 induced DLL4 expression, suggesting reciprocal inhibition between the 2 NOTCH ligands. However, silencing JAG1 or both JAG1 and DLL4 suppressed NICD, suggesting JAG1 is required for NICD induction in HLEC. These data indicate that mechanisms underlying deviant angiogenesis with $D L L 4$ deficiency are operational in fetal lung EC and are cell autonomous.

As DLL4 deficiency programs deviant vascularization, we posited that DLL4 is required for lung alveolarization. DLL4 was expressed in the alveolar septum of human lungs, and C57BL/6 and Dll4+/lacz mice, both at the base and leading EC of the alveolar septum. At E17.5, we noted that whereas distal lung acinar development was progressing in $D_{l l 4^{+/+}}$mice, there was an immature glandular structure in Dll $^{+/ l a c Z}$ littermates. At the early alveolar stage (P6), we observed double capillary loops in the primary septa of $D l l 4^{+/+}$mice; however, this was inconsistently observed in $D l l 4^{+/ l a c z}$ mice. Secondary septal development

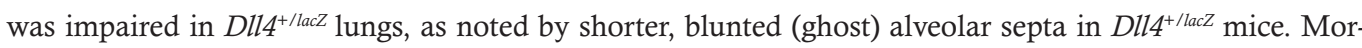
phometry revealed disrupted lung development from the saccular phase, and gender-independent impaired alveolarization in the mid-alveolar phase and on lung maturity in Dll4 $4^{+/ l a c Z}$ mice. Lung transcriptome profiling discovered that transcriptional regulators of AT1 maturation (Esr2 and $K l f 2$ ) progressively decreased in Dll4 ${ }^{+/ l a c Z}$ mice. Esr2 is highly expressed in both AT1 and AT2 cells, and Esr2-- mice have impaired alveolarization (38). KLF2 is a known regulator of AT1 and lung development (39). Gene expression of AT1 markers, such as Aqp5, Pdpn, and Hopx, decreased progressively during postnatal lung development in Dll4 ${ }^{+/ l a c Z}$ mice. AQP5 IF staining showed less continuously distributed AT1 in Dll4 ${ }^{+/ l a c Z}$ mice in comparison with $\mathrm{Dll}^{+/+}$mice, confirming defective AT1 development/maturation. Recent literature suggest that a diverse array of biological molecules secreted by EC facilitate organ development, i.e., "angiocrine signaling". Further investigation may lend clues to how D114-dependent EC secretion of angiocrine factors regulate AT1 development or maturation (40). Interestingly, targeting AT2 lineage showed that RNA, protein, and $\mathrm{SFTPC}^{+}$cells were increased with Dll4 haploinsufficiency. Similarly, PDGFRA ${ }^{+}$cells that mark myofibroblasts, and are critical for septal formation, were increased in Dll4 ${ }^{+/ l a c Z}$ mice (41). This was confirmed by increased protein and RNA expression in the postnatal lung. We speculate that PDGFRA ${ }^{+}$signaling induction represents a counterregulatory mechanism to compensate for impaired septal development with D114 hemizygosity. Although demonstrating the importance of EC DLL4 signaling to several lung lineages, it is outside the scope of the current study to investigate this complex biology.

This is the first study to our knowledge to demonstrate that DLL4 regulates microvascular growth and arborization during distal lung ontogeny, and Dll4 deficiency programs deviant angiogenesis disrupting lung vascular patterning and alveolarization. These phenotypic changes occurred concurrently with altered expression signatures of lung EC fate specification and Notch signaling, as well as 
impaired AT1 lineage marker expression. Studies employing drug-dependent VEGF-signaling blockade or VEGF rescue in experimental models of developing lung injury demonstrated the importance of VEGF in lung development $(4,42,43)$. These studies postulated "the vascular hypothesis," i.e., vascular injury in the developing lung programming defective alveolarization (4). Our results support the "vascular hypothesis" from a lung development perspective by indicating that D114, an EC Notch ligand, is obligately required for distal lung morphogenesis. We also demonstrate that hyperoxia, a major risk factor for BPD in premature infants, inhibits DLL4 expression in the developing mouse lung and human lung EC indicating a potential role for DLL4 in BPD. Future studies will examine crosstalk between EC DLL4 and other cell types required for distal lung development, and the relevance of deviant DLL4 signaling to impaired vascular development in BPD affecting preterm infants.

\section{Methods}

Antibodies and primers. Antibodies for staining: mouse anti-DLL4 (sc-365429, SCBT) and anti-PECAM (sc376764, SCBT); rabbit anti-ERG (ab92513, Abcam), anti-AQP5 (orb15125, Biorbyt), anti-APLNR (20341-1AP, Thermo Fisher Scientific), anti-SPC (ab90716, Abcam), anti-PDGFRA (ab203491, Abcam), anti-JAG1 (ab7771, Abcam); rat anti-PECAM (550274, BD Biosciences), anti-Ki67 (11-5698-82, Thermo Fisher Scientific); goat anti-Carbonic Anhydrase IV (CAR4) (PA5-47312, Thermo Fisher Scientific); and chicken anti- $\beta$ galactosidase (ab9361, Abcam). Antibodies for Western blot: mouse anti-DLL4 (sc-365429, SCBT), anti-HES1 (sc-166410, SCBT), anti-JAG1 (sc-390177, SCBT), anti-PECAM (sc-376764, SCBT), anti-NR2F2 (sc-271940, SCBT); rabbit anti-NICD (4147, Cell Signaling), anti-PDGFRA (ab203491, Abcam), anti-SPC (ab90716, Abcam), and anti-VEGFA (ab46154, Abcam); goat anti-DLL4 (ab7280, Abcam); anti-mouse $\beta$-actin (ACTB) (A1978, Sigma). The primers were purchased commercially from Sigma.

Mouse model. Care of mice during experimental procedures was conducted in accordance with the policies at the University of Missouri-Kansas City Lab Animal Resource Center (Kansas City, Missouri, USA) and the NIH guidelines for the care and use of laboratory animals. Protocols had prior approval from the University of Missouri-Kansas City IACUC. Dll4 ${ }^{+/ l a c z}$ CD1 $\left(\right.$ Dll4 $\left.^{+/-}\right)$mouse strain was rederived from cryofrozen sperm with permission from Janet Rossant (Department of Molecular and Medical Genetics, University of Toronto, Toronto, Canada). WT C57BL/6 and CD1 mice were obtained commercially from Charles River. Our experiments did not involve surgical procedures. Mice were euthanized using $100 \mathrm{mg} / \mathrm{kg}$ i.p injection pentobarbital, exsanguinated after cessation of heartbeat, and the lungs were harvested.

Cell culture and immortalized HLEC generation. Primary HLECs were purchased from ScienCell (HPMEC, 3000) and grown as described in previous studies (44). The lots used from ScienCell corresponded to fetal 18 -week-old male or female. We followed the company's protocol and immortalized HLEC with Lentivirus containing SV40 large T antigens (ABM). The in vitro angiogenesis network formation assay on Matrigel using primary HLECs was performed as previously described (45). 3D angiogenesis assay was performed as previously described (46). A total of $2.5 \mathrm{ng} / \mathrm{mL}$ VEGF was added but without fibroblasts. Beads coated with immortalized HLEC were embedded onto a 3D gel and cultured in 24-well plates for 4 days. A total of $1-4$ beads per well, 8 wells per group, were counted for 3D angiogenesis. HLECs were cultured in a sealed chamber containing $85 \% \mathrm{O}_{2}$ and $5 \% \mathrm{CO}_{2}$ for hyperoxia treatment.

Isolation of murine lung ECs. Mouse lung ECs were extracted from whole lung tissues as done previously (44).

siRNA-mediated gene silencing. siRNA sequences targeting human DLL4 (siDLL4) and JAG1 (siJAG1) were purchased from Santa Cruz Biotechnology (SCBT), and gene silencing was performed as per the manufacturer's recommendations (44). For the nonsilenced cells, control siRNA (consisting of scrambled sequence that does not interfere with cellular function) was used (SCBT).

shRNA-mediated DLL4 gene knockdown. For immortalized HLEC (HLEC-Im), lentivirus containing $D L L 4 \operatorname{shRNA}$ was generated and used to knockdown $D L L 4$ expression. For the nonsilenced cells, control shRNA that does not interfere with cellular function was used (Addgene).

Quantification of capillary diameter and intermediate blood vessels. For determining capillary diameter, we randomly chose 3 fields and measured 6 capillaries per field with Aperio ImageScope software (Leica Biosystems) across experimental groups ( $n=5$ in each group). For determining intermediate blood vessel numbers, we randomly chose 4 fields per mouse (sections) across experimental groups ( $n$ $=5$ in each group) and counted the number of blood vessels sized between 10 and $150 \mu \mathrm{m}$. The data were analyzed using 1-way ANOVA. 
IHC, IF, and X-gal staining. IHC and IF was done as in our previous study (44). The lungs of the mouse pups were fixed in glutaraldehyde and frozen, and sections were cut onto slides for X-gal staining. The frozen slides used for X-gal staining were fixed in glutaraldehyde for 10 minutes and then incubated in $\mathrm{X}$-gal staining buffer at $37^{\circ} \mathrm{C}$ for $1-2$ hours. The slides were fixed in formalin if IHC staining was needed after X-gal staining. The slides were counterstained in Nuclear Fast Red (Sigma) and mounted in Permount Mounting Medium. Human lung tissue from different developmental stages obtained from human autopsy specimens housed within the Pathology Department of Children's Mercy Hospital were used for IF staining. Whole-mount staining of E14.5 lung followed a published method (47). Primary antibodies are previously described. A sample size of $n \geq 3$ was used for the human and mouse sample/group.

Quantification of $\mathrm{SPC}^{+}, \mathrm{Ki} \mathrm{7}^{+}$, and PDGFRA $\mathrm{A}^{+}$cells. The sections of mouse lungs were prepared and stained as previously described. At least 5 mice were in each experimental group, and quantification of the percentage of SPC/DAPI, PDGFRA/Harris Hematoxylin, Ki67/ERG per high-power field (HPF) are reported as an average of 5 images taken per mouse.

Quantification of $m R N A$ expression using RT-PCR. Total RNA was extracted, and qRT-PCR was carried out as in the previous study (44). $\beta$-Actin and $18 S$ were used as housekeeping genes. The relative gene expression was calculated using the Pfaffl Method (48).

Immunoblotting for quantifying protein expression. Immunoblotting was carried out following standard protocols (44). Densitometry was performed using ImageJ Software (NIH), and changes were normalized to ACTB or the corresponding nonphosphorylated protein.

$H \& E$ staining to assess lung development in inflated mouse lungs. CD1 and C57B16 mouse pups were euthanized on P8, P14, and P28. The lungs were inflated using a fixed volume of formalin as previously described (44). Radial alveolar counts and mean linear intercepts were quantified as before (49). For P2 lungs, uninflated lungs were used for quantification of distal air space development.

RNA-Seq. Dll4 ${ }^{+/+}$and Dll4 ${ }^{+/ l a c}$ pups were euthanized using $100 \mathrm{mg} / \mathrm{kg}$ i.p injection pentobarbital at P6. Total cellular RNA was isolated from lungs using the MirVana kit (Thermo Fisher Scientific) according to the manufacturer's instructions. Poly-A selected NEBNext Ultra RNA Libraries (New England BioLabs) were sequenced on an Illumina NovaSeq platform using a 150-bp paired-end sequencing strategy (Novogene Corporation). Each sample was sequenced to an average of 52.8 million reads with $>94 \%$ of bases above Q30. Mapping of RNA-Seq reads and transcript assembly and abundance estimation were conducted and reported in Fragments Per Kilobase of exon per million fragments mapped (FPKM). To identify genes that were differentially expressed between heterozygous and WT mice, the FCs for each gene were calculated by dividing the average FPKM for the heterozygous mice by the average FPKM for the WT mice. Sample clustering was performed on log2-transformed FCs with the heatmap. $2 \mathrm{R}$ function from the R gplots package, using default parameters (Euclidian distance and "complete" clustering method). The differentially expressed genes were analyzed using Ingenuity Pathway Analysis (IPA, Ingenuity Systems Inc.). The data discussed in this publication have been deposited in NCBI's Gene Expression Omnibus (50) (GEO accession GSE164732; https://www.ncbi.nlm.nih.gov/geo/query/acc.cgi?acc=GSE164732).

scRNA-Seq and Dll4 expression plot. Three C57B16 pups were euthanized using $100 \mathrm{mg} / \mathrm{kg}$ i.p. injection pentobarbital at P14. Fresh lung single-cell digests furnished $10^{4}$ cells (19), which were loaded on a 10x Genomics v3 scRNA chip using $10 \times$ Genomics v3 scRNA reagents. Library preps were done, and completed libraries were sequenced on an Illumina NovaSeq 6000 instrument with 25,000 reads/cell targeted. Raw sequencing data were processed through bcl2fastq2 (Illumina), resulting in 2 FASTQ files per library. Individual 10× libraries were processed through Cell Ranger v3.0.2, and the resulting count matrices were imported into Seurat v3.1.0 for normalization and clustering following Seurat's SCTransform-based workflow. SingleR was used to assign a predicted cell type to each cluster. Dll4 expression was plotted for all cells from RA-exposed mice using the SCTransform normalized counts generated by Seurat. The data discussed in this publication have been deposited in NCBI's Gene Expression Omnibus (50) (GEO accession GSE164621; https://www.ncbi.nlm.nih.gov/geo/query/acc.cgi?acc=GSE164621).

Statistics. Data are shown as mean \pm SD or median with IQR, and a $P$ value of less than 0.05 was considered significant. For cell culture experiments, data from a minimum of 3 independent experiments with adequate technical replicates were used for quantification. All animal data were obtained in littermate controls. For animal experiments, a minimum of 5 animals were used for each experimental group. For histological quantification at least 2 slides with 3 replicates per mice were used. RNA quantification and PCR results had 2-3 technical replicates. We initially examined whether distribution of data was 
Gaussian using the D'Agostino-Pearson omnibus normality test. If data were normally distributed, then 1-way ANOVA with Tukey's post hoc test with multiple comparisons was used for analysis. For analysis with one comparison and normal distribution, the 2-tailed $t$ test with Welch's correction was used. If data did not meet Gaussian assumptions, a 2-tailed Mann-Whitney $U$ test was used for analysis. For most analysis, fold changes were calculated related to expression/changes in untreated controls. Statistical analysis was done using Graphpad Prism version 7.0.

Study approval. Lab experiments were reviewed and approved under the University of Missouri-Kansas City Institutional Biosafety Committee, protocol number 18-28. Animal experiments were reviewed and approved under the University of Missouri-Kansas City IACUC, protocol number 1510-02.

\section{Author contributions}

SX designed the project, conducted experiments, processed data, interpreted results, and wrote the manuscript. HLM prepared mice, performed immunoblotting and qRT-PCR, processed data, and prepared the figures. NT performed morphological analysis and DLL4 mapping. SM bred and genotyped mice, performed H\&E staining, and performed mouse lung morphological analysis. JJ analyzed scRNA-Seq data. DH analyzed Dll4 RNA-Seq data. MN and TK provided insight into certain aspects of study, helped with manuscript revision, and edited the manuscript. VS was responsible for overall conception of the project, including developing experimental design, interpreting results, and writing and editing the manuscript.

\section{Acknowledgments}

HLM, SX, and VS were supported by 1R01HL128374-01 (to VS). The authors thank Donald W. Thibeault, Children's Mercy Hospital, Kansas City (emeritus), for critical input and Wei Yu, Children's Mercy Hospital, Kansas City, for producing lentivirus vectors.

Address correspondence to: Venkatesh Sampath, Professor of Pediatrics, 2401 Gillham Rd., Division of Neonatology, Kansas City, Missouri 64108, USA. Phone: 816.234.3591; Email: vsampath@cmh.edu.

1. Schittny JC. Development of the lung. Cell Tissue Res. 2017;367(3):427-444.

2. Jobe AH, Bancalari E. NICHD/NHLBI/ORD workshop summary - bronchopulmonary dysplasia. Am J Respir Crit Care Med. 2001;163(7):1723-1729.

3. Morty RE. Recent advances in the pathogenesis of BPD. Semin Perinatol. 2018;42(7):404-412.

4. Thébaud B, Abman SH. Bronchopulmonary dysplasia: where have all the vessels gone? Roles of angiogenic growth factors in chronic lung disease. Am J Respir Crit Care Med. 2007;175(10):978-985.

5. Baker CD, Abman SH. Impaired pulmonary vascular development in bronchopulmonary dysplasia. Neonatology. 2015;107(4):344-351.

6. DeMello DE, et al. Early fetal development of lung vasculature. Am J Respir Cell Mol Biol. 1997;16(5):568-581

7. Galambos C, Demello DE. Molecular mechanisms of pulmonary vascular development. Pediatr Dev Pathol. 2007;10(1):1-17

8. Parera MC, et al. Distal angiogenesis: a new concept for lung vascular morphogenesis. Am J Physiol Lung Cell Mol Physiol. 2005;288(1):141-L149.

9. Hislop A. Developmental biology of the pulmonary circulation. Paediatr Respir Rev. 2005;6(1):35-43.

10. Blanco R, Gerhardt H. VEGF and notch in tip and stalk cell selection. Cold Spring Harb Perspect Med. 2013;3(1):1-19.

11. Duarte A, et al. Dosage-sensitive requirement for mouse D114 in artery development. Genes Dev. 2004;18(20):2474-2478.

12. Gale NW, et al. Haploinsufficiency of delta-like 4 ligand results in embryonic lethality due to major defects in arterial and vascular development. Proc Natl Acad Sci U S A. 2004;101(45):15949-15954.

13. Pitulescu ME, et al. Dl14 and Notch signalling couples sprouting angiogenesis and artery formation. Nat Cell Biol. 2017;19(8):915-927.

14. Trindade A, et al. Overexpression of delta-like 4 induces arterialization and attenuates vessel formation in developing mouse embryos. Blood. 2008;112(5):1720-1729.

15. Benedito R, et al. Loss of notch signalling induced by Dll4 causes arterial calibre reduction by increasing endothelial cell response to angiogenic stimuli. BMC Dev Biol. 2008;8:1-15.

16. Le Cras TD, et al. VEGF causes pulmonary hemorrhage, hemosiderosis, and air space enlargement in neonatal mice. Am $J$ Physiol Lung Cell Mol Physiol. 2004;287(1):L134-L142.

17. Kasahara Y, et al. Inhibition of VEGF receptors causes lung cell apoptosis and emphysema. J Clin Invest. 2000;106(11):1311-1319.

18. Ding BS, et al. Endothelial-derived angiocrine signals induce and sustain regenerative lung alveolarization. Cell. 2011;147(3):539-553.

19. Guo M, et al. Single cell RNA analysis identifies cellular heterogeneity and adaptive responses of the lung at birth. Nat Commun 2019;10(1):37.

20. Vila Ellis L, et al. Epithelial Vegfa specifies a distinct endothelial population in the mouse lung. Dev Cell. 2020;52(5):617-630.

21. Domigan CK et al. Autocrine VEGF maintains endothelial survival through regulation of metabolism and autophagy. J Cell Sci. 2015;128(12):2236-2248.

22. Rodríguez-castillo JA, et al. Understanding alveolarization to induce lung regeneration. Respir Res. 2018;19(1):148.

23. Lobov IB, et al. Delta-like ligand 4 (DII4) is induced by VEGF as a negative regulator of angiogenic sprouting. Proc Natl Acad 
Sci U S A. 2007;104(9):3219-3224.

24. MacK JJ, Luisa Iruela-Arispe M. NOTCH regulation of the endothelial cell phenotype. Curr Opin Hematol. 2018;25(3):212-218

25. Lobov I, Mikhailova N. The role of D114/Notch signaling in normal and pathological ocular angiogenesis: D114 controls blood vessel sprouting and vessel remodeling in normal and pathological conditions. J Ophthalmol. 2018;3565292.

26. Hellström M, et al. D114 signalling through notch1 regulates formation of tip cells during angiogenesis. Nature. 2007;445(7129):776-780.

27. Lobov IB, et al. The DII4/notch pathway controls postangiogenic blood vessel remodeling and regression by modulating vasoconstriction and blood flow. Blood. 2011;117(24):6728-6737.

28. Stevens M, Oltean S. Modulation of receptor tyrosine kinase activity through alternative splicing of ligands and receptors in the VEGF-A/VEGFR axis. Cells. 2019;8(4):288.

29. Benedito R, et al. The notch ligands D114 and Jagged1 have opposing effects on angiogenesis. Cell. 2009;137(6):1124-1135.

30. Johnson V, et al. Neurite mistargeting and inverse order of intraretinal vascular plexus formation precede subretinal vascularization in vldlr mutant mice. PLoS One. 2015;10(7):e0132013.

31. Muppala S, et al. Proangiogenic properties of thrombospondin-4. Arterioscler Thromb Vasc Biol. 2015;35(9):1975-1986.

32. Stamatovic SM, et al. CCL2 regulates angiogenesis via activation of Ets-1 transcription factor. J Immunol. 2006;177(4):2651-2661.

33. Wang X, et al. LRG1 promotes angiogenesis by modulating endothelial TGF- $\beta$ signalling. Nature. 2013;499(7458):306-311.

34. Huang S, et al. Stat1 negatively regulates angiogenesis, tumorigenicity and metastasis of tumor cells. Oncogene. 2002;21(16):2504-2512.

35. Zhang Y, et al. Overexpression of STAT1 suppresses angiogenesis under hypoxia by regulating VEGF-A in human glioma cells Biomed Pharmacother. 2018;104:566-575.

36. Bhattacharya $\mathrm{R}$, et al. Inhibition of vascular permeability factor/vascular endothelial growth factor-mediated angiogenesis by the Kruppel-like factor KLF2. J Biol Chem. 2005;280(32):28848-28851.

37. Numasaki M, et al. Interleukin-17 promotes angiogenesis and tumor growth. Blood. 2003;101(7):2620-2627.

38. Morani A, et al. Lung dysfunction causes systemic hypoxia in estrogen receptor beta knockout (ERbeta-/-) mice. Proc Natl Acad Sci U S A. 2006;103(18):7165-7169.

39. Pei L, et al. Thyroid hormone receptor repression is linked to type I pneumocyte-associated respiratory distress syndrome. Nat Med. 2011;17(11):1466-1472.

40. Rafii S, et al. Angiocrine functions of organ-specific endothelial cells. Nature. 2016;529(7586):316-325.

41. Li R, et al. Pdgfra marks a cellular lineage with distinct contributions to myofibroblasts in lung maturation and injury response. Elife. 2018;7:1-20.

42. Le Cras TD, et al. Treatment of newborn rats with a VEGF receptor inhibitor causes pulmonary hypertension and abnormal lung structure. Am J Physiol - Lung Cell Mol Physiol. 2002;283(3):555-562.

43. Thébaud B, et al. Vascular endothelial growth factor gene therapy increases survival, promotes lung angiogenesis, and prevents alveolar damage in hyperoxia-induced lung injury: evidence that angiogenesis participates in alveolarization. Circulation. 2005;112(16):2477-2486.

44. Menden $\mathrm{H}$, et al. Histone deacetylase 6 regulates endothelial MyD88-dependent canonical TLR signaling, lung inflammation, and alveolar remodeling in the developing lung. Am J Physiol Lung Cell Mol Physiol. 2019;317(3):L332-L346.

45. Xia S, et al. Endothelial immune activation programmes cell-fate decisions and angiogenesis by inducing angiogenesis regulator DLL4 through TLR4-ERK-FOXC2 signalling. J Physiol. 2018;596(8):1397-1417.

46. Nakatsu MN, Hughes CCW. Chapter 4 An optimized three-dimensional in vitro model for the analysis of angiogenesis. Methods Enzymol. 2008;443:65-82.

47. Metzger RJ, et al. The branching programme of mouse lung development. Nature. 2008;453(7196):745-750.

48. Pfaffl MW. A new mathematical model for relative quantification in real-time RT-PCR. Nucleic Acids Res. 2001;29(9):e45-e45.

49. Menden $\mathrm{H}$, et al. Lipopolysaccharide (LPS)-mediated angiopoietin-2-dependent autocrine angiogenesis is regulated by NADPH oxidase 2 (Nox2) in human pulmonary microvascular endothelial cells. J Biol Chem. 2015;290(9):5449-5461.

50. Edgar R, et al. Gene Expression Omnibus: NCBI gene expression and hybridization array data repository. Nucleic Acids Res. 2002;30(1):207-210 